\title{
Unruh effect for interacting particles with ultracold atoms
}

\author{
Arkadiusz Kosior $^{1,2}$, Maciej Lewenstein ${ }^{2,3}$, Alessio Celi ${ }^{2,4}$ * \\ 1 Instytut Fizyki imienia Mariana Smoluchowskiego, Uniwersytet Jagielloński, Łojasiewicza \\ 11, 30-348 Kraków, Poland \\ 2 ICFO-Institut de Ciències Fotòniques, The Barcelona Institute of Science and Technology, \\ 08860 Castelldefels (Barcelona), Spain \\ 3 ICREA - Pg. Lluís Companys 23, 08010 Barcelona, Spain \\ 4 Institute for Theoretical Physics, University of Innsbruck, and Institute for Quantum \\ Optics and Quantum Information, Austrian Academy of Sciences, Innsbruck, Austria \\ * alessio.celi@icfo.eu
}

May 13, 2022

\begin{abstract}
The Unruh effect is a quantum relativistic effect where the accelerated observer perceives the vacuum as a thermal state. Here we propose the experimental realization of the Unruh effect for interacting ultracold fermions in optical lattices by a sudden quench resulting in vacuum acceleration with varying interactions strengths in the real temperature background. We observe the inversion of statistics for the low lying excitations in the Wightman function as a result of competition between the spacetime and BCS Bogoliubov transformations. This paper opens up new perspectives for simulators of quantum gravity.
\end{abstract}

\section{Contents}

1 Introduction $\quad 2$

2 Derivation of discrete Dirac Hamiltonian 4

2.1 Non-interacting particles 4

2.1.1 Lagrangian density 4

2.1.2 Spin-connection and vielbein

2.1.3 Lagrangian density in the Rindler Universe 5

2.1.4 Dirac Hamiltonian 6

2.2 Interacting particles 7

2.2.1 Lagrangian density for interacting particles 7

2.2.2 Interacting Dirac Hamiltonian 8

3 Rindler Universe Dirac Fermions with attractive interactions 9

3.1 Self-consistent Minkowski Hamiltonian 9

3.2 The mean field Rindler Hamiltonian 11

3.3 Wightman function for an accelerated observer 12

3.4 Power spectrum at $T_{U} \approx 0$ and $T=0$ 
3.5 Power spectrum at $T_{U}>0$ and $T=0$

3.6 Power spectrum at $T_{U}>0$ and $T>0$

3.7 Experimental realization and detection of Unruh effect 19

4 Pairing function $\Delta(U, T) \quad$ 20

4.1 Solution on a cylinder 20

4.2 Solution on a torus 22

5 Conclusions and Outlook 2

A Analytic equation for $\Delta$ on torus 25

B Pairs density $\quad 26$

C Symmetries of $H_{k_{y}}^{M / R} \quad$ 26

References

\section{Introduction}

Since the seminal idea of Feynman [1] the capabilities and regimes of applications of quantum simulators [2,3 have rapidly grown in the last decades. In particular, systems naturally defined on a lattice like trapped ions [4,5] and neutral groundstate and Rydberg atoms in optical lattices and tweezers [6 8], are excellent platforms for simulating coursed-grained models. They are obviously well suited for simulating condensed matter phenomena where the lattice is intrinsically there. For instance, by engineering synthetic gauge fields by laser means $[9]$ in real or synthetic lattices $[10,11]$ one can experimentally realize Hofstadter model $[12,13]$ and visualize the edge states of quantum Hall effect [14-16], as well as realize other topological models like the Haldane model [17]. Similarly by lattice shaking [18], one can achieve nonAbelian gauge fields [19 20] and observe classical magnetism [21,22] (for comprehensive reviews see e.g. [23]). Such single-particle simulators are a first step towards experiments that could help solving open questions in many-body phenomena through quantum simulation, as for instance the relation between the Hubbard model and high- $\mathrm{T}_{c}$ superconductivity $\left.24-26\right]$. The combination of synthetic gauge fields with interactions could lead for instance to the realization of (quasi-)fractional quantum Hall states in ladders [27. 28] or quantum spin liquid [29] states in triangular lattices with ultracold atoms.

Lattice based atomic simulators are also well suited for simulating high-energy physics where the lattice is adopted as regularization tool for studying, e.g., strongly coupled gauge theories [30]. Lattice gauge theories can be interpreted as the natural next step after synthetic gauge fields, where the phases representing the classical gauge fields are promoted to operators acting on the gauge degrees of freedom living on the links of the lattice. By adopting convenient gauge invariant truncations of such degrees of freedom, one can design simulators of both Abelian [31 37] and non-Abelian gauge theories [38 40] capable to probe confinement, string breaking, and to study the dynamics of charges as in the first proof-of-principle experimental 
realization of the Schwinger model with four ions 41,42 . Together with the new classical simulation approach based on tensor network, e.g. 43 50], such simulators offer a new way to study challenging open questions in high-energy physics like the dynamics and the phase diagram of strong interactions at finite temperature and densities, for review see [51 [53]).

Recently, lattice based quantum simulators have been pushed forward also for the study of gravitational phenomena [54]. There is a natural and unresolved tension between quantum physics and general relativity that produces spectacular and counterintuitive effects. Indeed, the quantization requires the choice of a preferred time direction, which fixes the notions of vacuum state and particles, choice that it is at odd with general covariance and makes such notions depending on the observer [55, 56]. Such tension becomes manifest in presence of an event horizon and leads to the appearance of phenomena like the Hawking radiation of a black hole [57] and the Unruh effect [58] for an accelerated observer. The latter sees the vacuum as a thermal state with a temperature proportional to acceleration essentially because in his/her reference frame (Rindler metric) there is an event horizon and he/she can access only part of the vacuum [59 61. Simulating the Unruh effect and the Hawking radiation is interesting on one hand because these striking effects are hard to observe in nature, on the other hand because they are at the moment the only experimentally accessible phenomena that can bring us closer to quantum gravity.

There are essentially two ways of emulating gravity. The first known as analog gravity exploits the similarity between Navier-Stocks and Einstein-Hilbert equations to engineer the motion of classical and quantum fluids in artificial space-times (see 62 64 for a review). In particular the Unruh effect and the Hawking radiation were studied with phononic quasiparticles in a Bose-Einstein condensate 65 72, photons 73 76 and even classical surface waves on moving water 77,78 (for very recent ultracold atom experimental analogues of the cosmological expansion see [79,80]).

Alternatively, one can simulate the motion of artificial Dirac fermions in artificial gravity background by tailoring Dirac Hamiltonian on the lattice properly. This strategy allows for a systematic quantum simulation approach. As shown in [54] (see also [81 83]) one can engineer the motion of Dirac fermions in optical metrics [84 by making the Fermi velocity position dependent, that is by engineering position-dependent tunneling rates on the lattice, which can be equivalently translated in random walk processes $85-88$. One can apply such procedure to any lattice formulation of Dirac Hamiltonian [89], as for instance artificial graphene obtained from ultracold fermions in a brick-wall lattice [90 (see [91, 92 and 93 for realizations of hexagonal optical lattices). Note that one can obtain the motion of Dirac fermions in certain curved space-times by physically bending a graphene sheet 94,95$]$. In principle, one can observe signatures of the Unruh effect with some subtleties in such a system [96 98] as well as in more complicate scenarios [99.

Engineering artificial gravity through position-dependent tunneling rates for ultracold atoms in optical lattices offers several advantages, as we have shown recently in Ref. [100]:

1. It allows for much more tunability as it is as hard as engineering synthetic gauge fields (position dependent tunneling phases);

2. It allows for a direct experimental observation of the Unruh effect through a quantum quench;

3. It allows to go beyond single-particle phenomena and explore many-body physics in curved space-times. 
Here we take a first step in the this largely unexplored world and consider the manifestation of the Unruh effect for interacting fermions in two spatial dimensions. The model we consider is essentially equivalent to the 2D version of Thirring model [101] (the thermal nature of Hawking radiation was verified for $1 \mathrm{D}$ version in $[102]$ ). We propose an experimental protocol for studying the interplay between relativistic invariant quartic interactions and the acceleration with ultracold atoms. In the experiment, the acceleration of the (interacting) vacuum can be obtained by quenching the Dirac Hamiltonian as proposed first in [100], while the interactions can be controlled for instance by Feshbach resonance [103]. Alternatively, interaction can be mediated by another bosonic atom, as proposed for the Thirring model in flat space in [104. We show that when the system can be described thought BCS theory the Unruh effect occurs for the Cooper pairs: due to the "inversion of statics" [60] we observe a crossover between bosonic and fermionic thermal response in two spatial dimensions.

The paper is organized as follows. In Sec. 2 we review the derivation of the singleparticle and interacting naive Dirac Hamiltonian in the Rindler metric on a square lattice. The expert reader can directly to Sec. 3 that is the hearth of the paper where we discuss the theoretical and experimental aspects of the Unruh effect for interacting Dirac fermions. We consider the case of attractive interactions and derive the Wightman response functions in the mean field limit. We obtain it by determining the Bogoliubov transformation that relates the quasi-particle states in Rindler and Minkowski spacetime, once the normalization of the Rindler Hamiltonian relative to the Minkowski one is chosen such that the former equals the latter in the far horizon limit, i.e. on the boundaries of the cylindrical lattice. We find the expected thermal response function for the quasi-particles, in particular at low energy the effective description in terms of Cooper pairs translates in a Fermi-Dirac-like Planckian response characteristic of bosons. In Sec. 4 we take a step back and analyze in details the BCS theory for the naive Dirac Hamiltonian in Minkowski space. In particular, we discuss and explain its subtle dependence on the boundary conditions on finite-size systems (further explanation can be found in the Appedindix that contains also derivations relevant for Sec. 3). Finally, we draw our conclusions in Sec. 5, we resume our results and comment about the new perspectives and developments opened up by our work.

\section{Derivation of discrete Dirac Hamiltonian}

In this section, we present the derivation of the discrete Hamiltonian of interacting massless Dirac fermions in the Rindler Universe in $(2+1)$ dimensions. For the sake of clarity and to be self contained, we first review the derivation of the non-interacting Hamiltonian [54, 105], by starting from the general Lagrangian density for Dirac particles in $(d+1)$ curved spacetimes. We use this section also to fix the notion used in the following sections.

\subsection{Non-interacting particles}

\subsubsection{Lagrangian density}

The Lagrangian density of a massless non-interacting Dirac spinor $\psi$ in a $(d+1)$ dimensional curved spacetime reads (see, for example 106 108])

$$
\mathcal{L}_{0}=-i \bar{\psi} \gamma^{\mu} D_{\mu} \psi
$$


where $\bar{\psi}=\psi^{\dagger} \gamma^{0}$ and

$$
D_{\mu} \psi=\partial_{\mu} \psi+\frac{1}{4} w_{\mu}^{a b} \gamma_{a b} \psi
$$

Here $D_{\mu} \psi$ is the covariant derivative of a spinor $\psi$, introduced to guarantee the invariance under the local Poincaré transformations, $w_{\mu}^{a b}(x)$ is the spin-connection which gauges the Lorentz group, and $\gamma$ 's are the gamma matrices in a curved spacetime

$$
\left\{\gamma^{\mu}, \gamma^{\nu}\right\}=2 g^{\mu \nu}, \quad \gamma^{\mu \nu}=\frac{1}{2}\left[\gamma^{\mu}, \gamma^{\nu}\right], \quad \gamma_{\mu}=g_{\mu \nu} \gamma^{\nu}
$$

where $g^{\mu \nu}$ is the metric tensor $d s^{2}=g_{\mu \nu} d x^{\mu} d x^{\nu}$. Note that we are using the mostly-positive metric signature, where the norm of timelike vectors is negative. Choosing the opposite signature would require to multiply all gamma matrices by the imaginary unit and, as a consequence, changing sign of the Lagrangian density.

In $(2+1)$ dimensions $\gamma$ 's are $2 \times 2$ matrices, and can be expressed in terms of Pauli matrices. A possible choice for the "flat" gamma matrices in Minkowski space is

$$
\gamma_{0}=i \sigma_{z}, \quad \gamma_{1}=\sigma_{y}, \quad \gamma_{2}=-\sigma_{x}
$$

Irrespectively of the choice of the gamma matrices, in $(2+1)$ dimensions the product of all $\gamma$ 's is proportional to the identity

$$
\gamma_{0} \gamma_{1} \gamma_{2}=-1
$$

\subsubsection{Spin-connection and vielbein}

The relation between gamma matrices in curved spacetimes $\gamma_{\mu} \equiv \gamma_{\mu}(x)$ and the flat matrices $\gamma_{a}$ is given be a vielbein field $e_{\mu}^{a}$, i.e. $\gamma_{\mu}(x)=e_{\mu}^{a}(x) \gamma_{a}$. The vielbein field introduces locally flat Cartesian frame of reference. It is defined by

$$
e_{\mu}^{a}(x) \eta_{a b} e_{\nu}^{b}(x)=g_{\mu \nu}(x) \quad \text { or } \quad e_{a}^{\mu}(x) g_{\mu \nu} e_{b}^{\nu}(x)=\eta_{a b},
$$

where $e_{a}^{\mu}$ and $e_{\mu}^{a}$ are inverse matrices, and $e_{a}^{\mu} e_{\mu}^{b}=\delta_{a}^{b}$. Note that the vielbein $e_{\mu}^{a}$ and the spinconnection $w_{\mu}^{a b}(x)$ fields cannot be independent, as Lorentz translations must relate orthogonal frames in different points. The requirement of a covariantly constant vielbein [109] imposes

$$
D_{[\mu} e_{\nu]}^{a}=\partial_{[\mu} e_{\nu]}^{a}+w_{[\mu b}^{a} e_{\nu]}^{b}=0
$$

where the indices $\mu$ and $\nu$ are antisymmetrized. The spin-connection can be written explicitly using the Christoffel symbols $\Gamma_{\nu \rho}^{\mu}$

$$
\begin{aligned}
w_{\mu}^{a b} & =e_{\nu}^{a} \Gamma_{\sigma \mu}^{\nu} e^{\sigma b}-e^{\nu a} \partial_{\mu} e_{\nu}^{b} \\
\Gamma_{\nu \rho}^{\mu} & =\frac{1}{2} g^{\mu \alpha}\left(\partial_{\rho} g_{\alpha \nu}+\partial_{\nu} g_{\alpha \rho}-\partial_{\alpha} g_{\nu \rho}\right) .
\end{aligned}
$$

\subsubsection{Lagrangian density in the Rindler Universe}

We consider $(2+1)$ dimensional static metric of the Rindler Universe 60,61, 109

$$
d s^{2}=-x^{2} d t^{2}+d x^{2}+d y^{2}
$$


which describes a flat Minkowski spacetime from the point of view of an observed moving with the constant acceleration $a=1 /|x|$.

The Rindler universe is static. Indeed, with the above coordinate choice the Rindler metric (10) is manifestly time-independent, with the time-like Killing vector $B=\partial_{t}$. The manifest time-translational invariance allows to construct a conserved Hamiltonian function. We can obtain it in a simple manner from the Dirac Lagrangian in Rindler space we derive below.

From $(10)$, we choose the nonzero components of the vielbein as

$$
e_{t}^{0}=|x|, \quad e_{x}^{1}=1, \quad e_{y}^{2}=1,
$$

which produce a particularly transparent form of curved gamma matrices

$$
\gamma_{t}=|x| \gamma_{0}, \quad \gamma_{x}=\gamma_{1}, \quad \gamma_{y}=\gamma_{2} .
$$

Accordingly with the choice of a vielbien, we can construct the spin connection, which the only nonzero components are

$$
w_{t}^{01}=\frac{x}{|x|}=-w_{t}^{10}
$$

where the last equality comes from the antisymmetricity of $w_{\mu}^{a b}$ in its internal (flat) indices.

The Dirac Lagrangian density $\mathcal{L}_{0}$ written explicitly in the $(2+1)$ Rindler Universe becomes

$$
\mathcal{L}_{0}^{R}=-i \bar{\psi}\left(\gamma^{\mu} \partial_{\mu}-\frac{1}{2 x} \gamma_{0} \gamma_{2}\right) \psi
$$

\subsubsection{Dirac Hamiltonian}

The Hamiltonian density is obtained by the Legendre transformation

$$
\mathcal{H}=\frac{\delta \mathcal{L}}{\delta\left(\partial_{t} \psi\right)} \partial_{t} \psi-\mathcal{L}
$$

For the Dirac Lagrangian density $\mathcal{L}_{0}^{R}$ in the Rindler Universe we have

$$
\mathcal{H}_{0}^{R}=i \bar{\psi}\left(\gamma^{i} \partial_{i}-\frac{1}{2 x} \gamma_{0} \gamma_{2}\right) \psi
$$

Finally, we obtain the Rindler Hamiltonian by integrating the Hamiltonian density on a spacelike hypersurface $H_{0}^{R}=\int \mathrm{d} \Sigma \mathcal{H}_{0}^{R}$, where the volume element $\mathrm{d} \Sigma=\sqrt{-g} \mathrm{~d} x \mathrm{~d} y$ includes the metric determinant. It is very convenient to write the Hamiltonian in the fully symmetric form, i.e.,

$$
H_{0}^{R}=\frac{1}{2} \int \mathrm{d} \Sigma \mathcal{H}_{0}^{R}+\frac{1}{2} \int \mathrm{d} \Sigma\left(\mathcal{H}_{0}^{R}\right)^{\dagger},
$$

resulting in

$$
H_{0}^{R}=\frac{i}{2} \int \mathrm{d} x \mathrm{~d} y|x|\left(\sigma_{x} \partial_{x} \psi^{\dagger}+\sigma_{y} \partial_{y} \psi^{\dagger}\right) \psi+\text { H.c. },
$$

where we express the $\gamma$ 's in terms of the Pauli matrices (4). 
Once written in the form of (18), we can properly discretize the Rindler Hamiltonian simply by replacing integration $\int \mathrm{d} x \mathrm{~d} y$ with summation $d^{2} \sum_{m, n}$, where $d$ is the lattice spacing, and by replacing the derivatives with finite differences

$$
\begin{aligned}
f \partial_{x} h & \rightarrow \frac{f_{m+1, n}+f_{m, n}}{2} \frac{h_{m+1, n}-h_{m, n}}{d}, \\
f \partial_{y} h & \rightarrow \frac{f_{m, n+1}+f_{m, n}}{2} \frac{h_{m, n+1}-h_{m, n}}{d},
\end{aligned}
$$

which fulfill the Leibnitz rule for differentiation. Finally, the Rindler Hamiltonian on the lattice reads

$$
H_{0}^{R}=i \sum_{m, n}\left(t_{m}^{x} \psi_{m+1, n}^{\dagger} \sigma_{x} \psi_{m, n}+t_{m}^{y} \psi_{m, n+1}^{\dagger} \sigma_{y} \psi_{m, n}\right)+\text { H.c. }
$$

which has the form of a Hubbard Hamiltonian with non-Abelian non-uniform tunneling terms

$$
t_{m}^{x}=\frac{|m+1|+|m|}{2}, \quad t_{m}^{y}=|m| .
$$

Note that the Rindler Hamiltonian is scale invariant, as it does not depend on the lattice spacing $d$ ( $d^{2}$ from the volume element cancels due to the replacement $\psi_{m, n} \rightarrow d^{-1} \psi_{m, n}$, done to guarantee the normalization condition $\int \mathrm{d} x \mathrm{~d} y \psi^{\dagger} \psi=1$ ).

A very similar derivation can be performed to obtain the Dirac Hamiltonian in a flat Minkowski space. In fact, since the Rindler Hamiltonian in the symmetric form (18) does not contain spin connection terms, it suffices to replace the metric determinant with a constant, i.e. $\sqrt{-g}=c$, which consequently leads to a discrete Hubbard Hamiltonian with a constant tunneling $t$ (hereafter we choose $t$ as an energy scale, i.e. we put $t=1$ ).

$$
H_{0}^{M}=i \sum_{m, n}\left(\psi_{m+1, n}^{\dagger} \sigma_{x} \psi_{m, n}+\psi_{m, n+1}^{\dagger} \sigma_{y} \psi_{m, n}\right)+\text { H.c. . }
$$

The Minkowski Hamiltonian (23) should be recovered from the Rindler Hamiltonian (21) in the asymptotic limit of small acceleration $a=\lim _{|m| \rightarrow \infty} 1 /|m|=0$. Therefore, we should choose a common energy scale at the boundary of the system. This is done by rescaling the tunneling values 22

$$
t_{m}^{x} \rightarrow t_{m}^{\prime}=\frac{|m+1|+|m|}{2 M}, \quad t_{m}^{y} \rightarrow t_{m}^{y}=\frac{|m|}{M},
$$

where $m=-M, \ldots, M$.

\subsection{Interacting particles}

\subsubsection{Lagrangian density for interacting particles}

In order to describe interacting Dirac particles we need to include a nonlinear term in the Lagrangian density (14)

$$
\mathcal{L}=\mathcal{L}_{0}+\mathcal{L}_{\text {int }} .
$$

The simplest choices for $\mathcal{L}_{\text {int }}$, which would guarantee Lorentz invariance and U(1)-current conservation are 
- density-density interaction 110

$$
\mathcal{L}_{\text {int }}=-\frac{\lambda}{2}(\bar{\psi} \psi)^{2}
$$

- current-current interaction 101, 111

$$
\mathcal{L}_{\text {int }}=\frac{\lambda}{2}\left(\bar{\psi} \gamma^{\mu} \psi\right)\left(\bar{\psi} \gamma_{\mu} \psi\right)
$$

Note that in $(3+1)$ spacetime dimensions, other possible choices would be $\mathcal{L}_{\text {int }}=\frac{\lambda}{2}\left(\bar{\psi} \gamma_{5} \psi\right)^{2}$ or $\mathcal{L}_{\text {int }}=\frac{\lambda}{2}\left(\bar{\psi} \gamma_{5} \gamma^{\mu} \psi\right)\left(\bar{\psi} \gamma_{5} \gamma_{\mu} \psi\right) \mid 112$, but in $(2+1)$ dimensions a $\gamma_{5}$ matrix is trivial - the product of all gamma matrices is proportional to the identity (5), reflecting the well-known fact that chirality is absent in even spatial dimensions.

\subsubsection{Interacting Dirac Hamiltonian}

The Legendre transformation of the full Lagrangian density (25) leads to the Hamiltonian density

$$
\mathcal{H}=\mathcal{H}_{0}-\mathcal{L}_{\text {int }} .
$$

Similarly to the non-interacting case, one gets the full Hamiltonian by integration over a spacelike hypersurface

$$
H=\int \mathrm{d} \Sigma \mathcal{H}=H_{0}+H_{\text {int }}, \quad H_{\text {int }}=-\int \mathrm{d} \Sigma \mathcal{L}_{\text {int }} .
$$

The discretized version of 29 is straightforward, as neither choice of the Lagrangian interaction density (26) - 27) includes derivatives. For the Rindler Universe and densitydensity interactions (27), we obtain

$$
H_{\text {int }}^{R}=\frac{\lambda}{2} \int \mathrm{d} \Sigma(\bar{\psi} \psi)^{2}=\sum_{m, n} U_{m} n_{\uparrow, m, n} n_{\downarrow, m, n}-\sum_{m, n} \frac{U_{m}}{2} n_{m, n},
$$

where we decompose the lattice spinor operator $\psi_{m, n}=\left[c_{\uparrow, m, n} c_{\downarrow, m, n}\right]$, introduce the number operators $n_{\sigma, m, n}=c_{\sigma, m, n}^{\dagger} c_{\sigma, m, n}, n_{m, n}=\sum_{\sigma} n_{\sigma, m, n}$, and define $U_{m}=|m| \lambda / d \equiv|m| U$.

Also, we can easily check that the other choice of Lorentz-invariant interactions (27) leads to an equivalent term

$$
-\frac{\lambda}{2} \int \mathrm{d} \Sigma\left(\bar{\psi} \gamma^{\mu} \psi\right)\left(\bar{\psi} \gamma_{\mu} \psi\right)=3 \sum_{m, n} U_{m} n_{\uparrow, m, n} n_{\downarrow, m, n}-\sum_{m, n} \frac{U_{m}}{2} n_{m, n},
$$

as apart from a different coefficient multiplying $n_{\uparrow, m, n} n_{\downarrow, m, n}$, both interaction terms in $(2+1)$ dimensions have the same form.

Finally, let us compare (30) with the corresponding interaction Hamiltonian in the Minkowski space

$$
H_{i n t}^{M}=U \sum_{m, n} n_{\uparrow, m, n} n_{\downarrow, m, n}-\frac{U}{2} \sum_{m, n} n_{m, n} .
$$

In order to make the two terms to coincide in the limit of zero acceleration we have to renormalize $U_{m}$ accordingly to

$$
U_{m} \rightarrow U_{m}^{\prime}=U_{m} / M
$$

such to make the value of the couplings to coincide at the lattice boundaries $U_{M}^{\prime}=U$ as previously done in the non-interacting case for the tunneling, $t_{M}^{\prime x, y} \sim t=1$. 


\section{Rindler Universe Dirac Fermions with attractive interactions}

In this section we consider a model of Dirac fermions with attractive interactions in the meanfield regime. The primary focus of this section is to analyze the power spectrum of the Rindler noise with increasing interaction strength, and show the difference between interacting thermal particles in a flat space and interacting accelerating particles at non-zero Unruh temperature $T_{U}$. In order to compute it we first find quasi-particle basis in the Minkowski and in the Rindler space for appropriately normalized Hamiltonians defined in the previous section. Indeed, Rindler Hamiltonian has to coincide with the Minkowski one in the limit of zero acceleration. On the lattice, this requirement translates in matching the coupling on the boundaries in $x$ (we exploit the translational invariance along $y$ by taking it periodic such that our lattice is a cylinder). Then, we find the unitary transformation between the two quasi-particle basis and determine the Wightman function on the Minkowski ground state in the Rindler universe, i.e., as measured after quenching the Hamiltonian from Minkowski to Rindler spacetime. Finally, we study the case in which the Minkowski background in which the observer accelerate is the thermal state of the interacting Dirac Hamiltonian. We conclude the section by discussing the experimental requirements for observing the Unruh effect in presence of interactions with ultracold fermions in optical lattices.

\subsection{Self-consistent Minkowski Hamiltonian}

Let us start with writing effective mean-field Hamiltonian in the Minkowski spacetime at $T=0$ and express eigensolutions in terms of quasiparticle modes.

The full Hamiltonian of interacting Dirac particles in the Minkowski spacetime is a sum of noninteracting (23) and interacting (32) terms

$$
H^{M}=H_{0}^{M}+H_{\text {int }}^{M},
$$

or explicitly in $(2+1)$ dimensions

$$
H^{M}=i \sum_{m, n}\left(\psi_{m+1, n}^{\dagger} \sigma_{x} \psi_{m, n}+\psi_{m, n+1}^{\dagger} \sigma_{y} \psi_{m, n}\right)+\text { H.c. }+U \sum_{m, n} n_{\uparrow, m, n} n_{\downarrow, m, n}-\frac{U}{2} \sum_{m, n} n_{m, n},
$$

where $U$ is an attractive interaction strength $U=-|U|$, and $\psi_{m, n}=\left[c_{\uparrow, m, n} c_{\downarrow, m, n}\right], n_{\sigma, m, n}=$ $c_{\sigma, m, n}^{\dagger} c_{\sigma, m, n}, n_{m, n}=\sum_{\sigma} n_{\sigma, m, n}$. We tackle the problem by approximating the interaction term with a mean field averages 113 114

$$
U n_{\uparrow, m, n} n_{\downarrow, m, n} \approx\left(\Delta c_{\uparrow, m, n}^{\dagger} c_{\downarrow, m, n}^{\dagger}+\Lambda c_{\uparrow, m, n}^{\dagger} c_{\downarrow, m, n}+\text { H.c. }\right)+W n_{m, n},
$$

where we consider all possible terms, i.e.

$$
\begin{aligned}
\Delta & =-U\left\langle c_{\uparrow, m, n} c_{\downarrow, m, n}\right\rangle, \\
W & =U\left\langle n_{\sigma, m, n}\right\rangle, \quad \sigma=\uparrow, \downarrow, \\
\Lambda & =U\left\langle c_{\downarrow, m, n}^{\dagger} c_{\uparrow, m, n}\right\rangle .
\end{aligned}
$$

Notice that (39) does not conserve spin (or species) of particles and therefore, we expect it to be identically zero $\Lambda \equiv 0$. Consequently, the mean field Minkowski Hamiltonian for interacting particles is

$$
H_{m f}^{M}=H_{0}^{M}+H_{n}^{M}+H_{\Delta}^{M}
$$


where $H_{0}^{M}$ is a free particle term given by $(23), H_{n}^{M}$ is an on-site potential energy term (where we include the chemical potential $\mu$ )

$$
H_{n}^{M}=\sum_{m, n}\left(W-\frac{U}{2}-\mu\right) \psi_{m, n}^{\dagger} \psi_{m, n},
$$

and $H_{\Delta}^{M}$ is a pairing term

$$
H_{\Delta}^{M}=\Delta \sum_{m, n} c_{\uparrow, m, n}^{\dagger} c_{\downarrow, m, n}^{\dagger}+\text { H.c. }=\frac{i \Delta}{2} \sum_{m, n} \psi_{m, n}^{\dagger} \sigma_{y} \psi_{m, n}^{*}+\text { H.c. . }
$$

Before we proceed to the eigensolutions of (40), let us stress that $\Delta$ and $W$ are given in (37) - (38), where the averages are calculated in the ground state $|0\rangle_{M}$ of $H_{m f}^{M}$. Consequently, $\Delta$ and $W$ are not external parameters and the eigenvalue problem should be solved self-consistently. Furthermore, since both Minkowski and Rindler metrics are translation invariant in $y$ direction, we consider the eigenvalue problem on the cylinder (see Appendix A for the analytical solution on a torus in Minkowski space). After performing the Fourier transformation $\psi_{m, n}=1 / \sqrt{N_{y}} \sum_{k_{y}} e^{i k_{y} n} \psi_{m, k_{y}}$, we write the Hamiltonian in a compact matrix form

$$
H_{m f}^{M}=\frac{1}{2} \sum_{k_{y}} \Psi_{k_{y}}^{\dagger} H_{k_{y}}^{M} \Psi_{k_{y}}
$$

where a spinor $\Psi_{k_{y}}$ is defined as

$$
\Psi_{k_{y}}^{\dagger}=\left(\cdots \psi_{m, k_{y}}^{\dagger} \psi_{m+1, k_{y}}^{\dagger} \cdots \psi_{m,-k_{y}}^{T} \psi_{m+1,-k_{y}}^{T} \cdots\right)
$$

and

$$
H_{k_{y}}^{M}=\left(\begin{array}{cc}
\sigma_{x} P_{x}+\epsilon_{k_{y}} \sigma_{y} & i \Delta \sigma_{y} \\
-i \Delta^{*} \sigma_{y} & \sigma_{x} P_{x}-\epsilon_{k_{y}} \sigma_{y}
\end{array}\right),
$$

where $P_{x}$ is a discrete derivate in $x$ direction $P_{x} \psi_{m, k_{y}}=-i t\left(\psi_{m+1, k_{y}}-\psi_{m-1, k_{y}}\right)$ and $\epsilon_{k_{y}}=$ $2 t \sin k_{y}$. In the Hamiltonian matrix (45) we drop the on-site potential term 41], as we choose the half-filling condition which yields $W=U / 2$ at $\mu=0$.

Eventually, we can express the spinor $\Psi_{k_{y}}$ in terms of the quasiparticle eigenmodes

$$
\begin{array}{r}
\Psi_{k_{y}}=\sum_{p}^{\prime}\left\{\left(\begin{array}{c}
U_{k_{y}, p}^{M} \\
V_{k_{y}, p}^{M}
\end{array}\right) \beta_{k_{y}, p}^{M}+\left(\begin{array}{c}
V_{-k_{y}, p}^{M *} \\
U_{-k_{y}, p}^{M *}
\end{array}\right) \beta_{-k_{y}, p}^{M \dagger}\right\}=\sum_{p}^{\prime}\left(\begin{array}{cc}
U_{k_{y}, p}^{M} & V_{-k_{y}, p}^{M *} \\
V_{k_{y}, p}^{M} & U_{-k_{y}, p}^{M *}
\end{array}\right)\left(\begin{array}{c}
\beta_{k_{y}, p}^{M} \\
\beta_{-k_{y}, p}^{M \dagger}
\end{array}\right) \\
\equiv \sum_{p}^{\prime} M_{k_{y}, p}\left(\begin{array}{c}
\beta_{k_{y}, p}^{M} \\
\beta_{-k_{y}, p}^{M \dagger}
\end{array}\right),
\end{array}
$$

where $\sum_{p}^{\prime}$ is a summation over positive eigenenergies of the matrix Hamiltonian 45 labeled by $p, E_{k_{y}, p}^{M} \geq 0$, and

$$
X_{k_{y}, p}^{(+)}=\left(\begin{array}{c}
U_{k_{y}, p}^{M} \\
V_{k y, p}^{M}
\end{array}\right), \quad X_{k_{y}, p}^{(-)}=\left(\begin{array}{c}
V_{-k_{y}, p}^{M *} \\
U_{-k y, p}^{M *}
\end{array}\right)
$$

are column eigenvectors to $E_{k_{y}, p}^{M}$ and $-E_{k_{y}, p}^{M}$, respectively (see Appendix A.

The expression (46) is a canonical Bogoliubov transformation between interacting particles and quasiparticles which diagonalize the Hamiltonian (45). 


\subsection{The mean field Rindler Hamiltonian}

In the Sec. 2 we derive a kinetic (21) and interacting (29) terms of the Dirac Hamiltonian in the Rindler Universe $H^{R}=H_{0}^{R}+H_{i n t}^{R}$, explicitly

$$
\begin{array}{r}
H^{R}=i \sum_{m, n}\left(t_{m}^{\prime} \psi_{m+1, n}^{\dagger} \sigma_{x} \psi_{m, n}+t_{m}^{\prime y} \psi_{m, n+1}^{\dagger} \sigma_{y} \psi_{m, n}\right)+\text { H.c. } \\
+\sum_{m, n} U_{m}^{\prime} n_{\uparrow, m, n} n_{\downarrow, m, n}-\sum_{m, n} \frac{U_{m}^{\prime}}{2} n_{m, n},
\end{array}
$$

where primes denote the rescaled tunnelings (24) and interactions (33).

Let us stress again that an accelerated observes moves in the physical vacuum $|\Omega\rangle$, which is a ground state of the Minkowski Hamiltonian $|\Omega\rangle=|0\rangle_{M}$. Although the Rindler Hamiltonian governs the dynamics of an accelerated observer, its ground state $|0\rangle_{R}$ does not obviously need to coincide with the physical vacuum $|0\rangle_{R} \neq|\Omega\rangle$. Therefore, from the point of an accelerated observed, its background is an excited state.

For that reason, the mean field averages for the interaction terms should now be calculated not in the ground state $|0\rangle_{R}$, but in the physical vacuum $|\Omega\rangle=|0\rangle_{M}$. For example, the Rindler pairing function $\Delta_{R}$ reads

$$
\Delta_{R}(m)=-U_{m}^{\prime}\left\langle c_{\uparrow, m, n} c_{\downarrow, m, n}\right\rangle=\xi_{m} \Delta,
$$

where $\xi_{m}=|m| / M$ is a rescaled distance from the horizon.

Apart from the position-dependent elements, the Rindler Hamiltonian (48) has the same form as the Minkowski Hamiltonian (35), and therefore we obtain its mean field counterpart practically automatically

$$
H_{m f}^{R}=\frac{1}{2} \sum_{k_{y}} \Psi_{k_{y}}^{\dagger} H_{k_{y}}^{R} \Psi_{k_{y}}
$$

with

$$
H_{k_{y}}^{R}=\left(\begin{array}{cc}
\sigma_{x} R(\xi)+\epsilon_{k_{y}}(\xi) \sigma_{y} & i \Delta(\xi) \sigma_{y} \\
-i \Delta^{*}(\xi) \sigma_{y} & \sigma_{x} R(\xi)-\epsilon_{k_{y}}(\xi) \sigma_{y}
\end{array}\right),
$$

where $\xi$ is a rescaled position operator $\xi \psi_{m, k_{y}}=\xi_{m} \psi_{m, k_{y}}, \Delta(\xi)=\xi \Delta$ is a position-dependent pairing function, $\epsilon_{k_{y}}(\xi)=\xi \epsilon_{k_{y}}$ a position-dependent tunneling energy (transverse to the horizon), and $R(\xi)$ is a discrete $|x| P_{x}$ operator $R(\xi) \psi_{m, k_{y}}=-i t_{m}^{\prime x} \psi_{m+1, k_{y}}+i t_{m-1}^{\prime x} \psi_{m-1, k_{y}}$.

Once solving the eigenvalue equation for $H_{k_{y}}^{R}$, one can express the spinor $\Psi_{k_{y}}$ (44) in terms of the Rindler quasiparticles

$$
\Psi_{k_{y}}=\sum_{p}^{\prime}\left(\begin{array}{cc}
U_{k_{y}, p}^{R} & V_{-k_{y}, p}^{R *} \\
V_{k_{y}, p}^{R} & U_{-k_{y}, p}^{R *}
\end{array}\right)\left(\begin{array}{c}
\beta_{k_{y}, p}^{R} \\
\beta_{-k_{y}, p}^{R \dagger}
\end{array}\right) \equiv \sum_{p}^{\prime} R_{k_{y}, p}\left(\begin{array}{c}
\beta_{k y, p}^{R} \\
\beta_{-k_{y}, p}^{R \dagger}
\end{array}\right),
$$

where we remind the reader that the prime in summation indicates that $p$ in $\sum_{p}^{\prime}$ runs over the positive eigenvalue, $E_{k_{y}, p}^{R}>0$.

Now, let us focus on how the Unruh temperature $T_{U}$ influences the Rindler pairing $\Delta_{R}$ (in Sec. 4 we discuss how the pairing $\Delta$ in the Minkowski space changes with the physical temperature $T)$. Since $\Delta_{R}(m)=\xi_{m} \Delta$ and $\xi_{m}$ is proportional to the inverse of acceleration $1 / a=|m|$, we could be tempt to write that the $\Delta_{R}$ is proportional to the inverse of Unruh 
temperature $T_{U}=a /(2 \pi)$. Nevertheless such conclusion would not be correct. The Unruh temperature is defined locally on $x=$ constant-hypersurfaces of the Rindler Universe and therefore is different for inequivalent observers. The expression (49) tells us how the pairing function changes with $|m|$ from the point of view of the Minkowski observer. Since the proper time slows down the closer we are to the horizon, then the interactions seem to be weaker. Therefore $\Delta_{R}(m)$ for the observer at $|m|$ should be rescaled with the proper time $\Delta_{R}(m) / \xi_{m}=$ $\Delta$. Consequently, the pairing strength seen by an accelerated observer does not depend on the Unruh temperature. At the same time, an observer at $|m|$ sees the pairing to be weaker $\Delta_{R}(m) / \xi_{m^{\prime}}<\Delta$ when she/he looks towards the horizon and stronger $\Delta_{R}(m) / \xi_{m^{\prime}}>\Delta$ when she/he looks opposite to the horizon.

\subsection{Wightman function for an accelerated observer}

The expressions (46) and (52) are canonical Bogoliubov transformations [113, 114 between interacting fermionic particles and noninteracting quasiparticles in the Minkowski and Rindler spacetimes, respectively. As quasiparticles modes (46) - (52) diagonalize the mean-field Hamiltonian 443$)$ - (50), we can write

$$
\begin{array}{r}
H_{m f}^{A}=\frac{1}{2} \sum_{k_{y}} \Psi_{k_{y}}^{\dagger} H_{k_{y}}^{A} \Psi_{k_{y}}=\frac{1}{2} \sum_{k_{y}} \sum_{p}^{\prime} E_{k_{y}, p}^{A}\left(\beta_{k_{y}, p}^{A \dagger} \beta_{k_{y}, p}^{A}-\beta_{-k_{y}, p}^{A} \beta_{-k_{y}, p}^{A \dagger}\right)= \\
\sum_{k_{y}} \sum_{p}^{\prime} E_{k_{y}, p}^{A} \beta_{k_{y}, p}^{A \dagger} \beta_{k_{y}, p}^{A}+\text { const. },
\end{array}
$$

where $p$ in the sum runs over positive eigenvalues and $A$ refers to either Minkowski $(M)$ or Rindler $(R)$. Note that in the last equality of 53 we use the fact that $H_{ \pm k_{y}}^{A}$ have the same spectra (see the discussion of the symmetries of $H_{k_{y}}^{A}$ in Appendix C).

From (53) we see that the groundstate of $H_{m f}^{A}$ is deprived of quasiparticle excitations. Therefore, it is anihilated by all quasiparticle operators

$$
\beta_{k_{y}, p}^{A}\left|0_{A}\right\rangle=0, \quad \forall k_{y}, p \text { s.t. } E_{k_{y}, p}^{A}>0 .
$$

A state that fulfills (54) is of a form

$$
\left|0_{A}\right\rangle \propto \prod_{k_{y}, p, E_{k_{y}, p}^{A}<0} \beta_{k_{y}, p}^{A \dagger}|0\rangle,
$$

where $|0\rangle$ where is a particle vacuum. Since quasiparticle operators mix particle creation and annihilation processes, we directly see that the ground state $\left|0_{A}\right\rangle$ must contain particles, and that the groundstates of Minkowski and Rindler Hamiltonians are different as they have different quasiparticle excitations. Also, because quasiparticle modes (46) and (52) are different for the two (stationary and accelerated) observers, the act of creation of a particle is seen differently. Combining (46) and (52) together and using $R_{k_{y}, p}^{\dagger} R_{k_{y}^{\prime}, p^{\prime}}=\delta_{k_{y}, k_{y}^{\prime}} \delta_{p, p^{\prime}}$ we obtain

$$
\left(\begin{array}{c}
\beta_{k_{y}, p}^{R} \\
\beta_{-k_{y}, p}^{R \dagger}
\end{array}\right)=\sum_{p^{\prime}}^{\prime} R_{k_{y}, p}^{\dagger} M_{k_{y}, p^{\prime}}\left(\begin{array}{c}
\beta_{k_{y}, p^{\prime}}^{M} \\
\beta_{-k_{y}, p^{\prime}}^{M \dagger}
\end{array}\right),
$$

which is the spacetime Bogoliubov transformation [60,61 between two noninteracting quasiparticles from the point of view of different observers. It is straightforward to find out that in 
general $\beta_{k_{y}, p}^{R}\left|0_{M}\right\rangle \neq 0$, and so indeed $\left|0_{M}\right\rangle \neq\left|0_{R}\right\rangle$. In particular, we expect a different response from a particle detector for different observers. Let us write the Wightman function for an accelerated observer

$$
G_{m, n}(t)=\left\langle 0_{M}\left|c_{\sigma, m, n}^{\dagger}(t) c_{\sigma, m, n}\right| 0_{M}\right\rangle,
$$

and its Fourier time transform

$$
G_{m, n}(\omega)=\int \mathrm{d} t e^{-i \omega t} G_{m, n}(t)
$$

which is the power spectrum of the Rindler noise.

Because the time evolution $c_{\sigma, m, n}^{\dagger}(t)$ is different for the stationary and accelerated observers, and since $\left|0_{M}\right\rangle \neq\left|0_{M}\right\rangle$, it is intuitive that a response function $G_{m, n}(\omega)$ should also be different for the two reference frames. However, the most interesting part is far less intuitive: (i) the response function $G_{m, n}(\omega)$ for an accelerated observed exhibits thermal behavior, (ii) in even spacetime dimensions thermal distribution of fermions (bosons) is Fermi-Dirac (Bose-Einstein), but in odd spacetime dimensions the statistic interchange.

In particular, in the continuous limit the power spectrum of a thermal noninteracting gas in $(2+1)$ Minkowski spacetime is [60]

$$
G_{M}^{T}(\omega)=\left\{\begin{array}{cc}
|\omega|\left|e^{\omega / T}+1\right|^{-1} & \text { (fermions) } \\
\left|e^{\omega / T}-1\right|^{-1} & \text { (bosons) }
\end{array}\right.
$$

whereas an accelerated observer sees

$$
G(\omega)=\left\{\begin{array}{cc}
|\omega|\left|e^{\omega / T_{U}}-1\right|^{-1} & \text { (fermions) } \\
\left|e^{\omega / T_{U}}+1\right|^{-1} & \text { (bosons) }
\end{array}\right.
$$

where the Unruh temperature is $T_{U}=a /(2 \pi)$.

One might wonder how to relate the fermionic and bosonic power spectra of a cold thermal gas $(T \rightarrow 0)$ in a flat space, to the ones seen by an accelerated observer in the limit of zero acceleration $\left(T_{U} \rightarrow 0\right)$. In fact, it is easy to find out that in the zero temperature limit, the modulus of the Bose distribution is Fermi-Dirac

$$
\lim _{T \rightarrow 0}\left|e^{\omega / T}-1\right|^{-1}=\left\{\begin{array}{cc}
0 & , \omega>0 \\
1 / 2 & , \omega<0
\end{array},\right.
$$

therefore, except for a singular point at $\omega=0$, the power spectra match exactly in the zero temperature limit.

Applying the spacetime Bogoliubov transformation (56) we get explicitly the Wightman function

$$
G_{m}(t) \equiv G_{m, n}(t)=\frac{1}{N_{y}} \sum_{k_{y}} \sum_{p, p^{\prime}}^{\prime}\left(u_{\sigma, m, k_{y}, p}^{R *}(t) \Gamma_{k_{y}, p, p^{\prime}}^{(1)}++v_{\sigma, m,-k_{y}, p}^{R}(t) \Gamma_{k_{y}, p, p^{\prime}}^{(2)}\right) v_{\sigma, m,-k_{y}, p^{\prime}}^{M *}
$$

where $u_{\sigma, m, k_{y}, p}^{R / M}$ and $v_{\sigma, m, k_{y}, p}^{R / M}$ are the elements of column vectors $U_{k_{y}, p}^{R / M}$ and $V_{k_{y}, p}^{R / M}$, respectively,

$$
\begin{aligned}
& u_{\sigma, m, k_{y}, p}^{R}(t)=e^{-i E_{k_{y}, p}^{R} t} u_{\sigma, m, k_{y}, p}^{R}, \\
& v_{\sigma, m, k_{y}, p}^{R}(t)=e^{-i E_{k_{y}, p}^{R} t} v_{\sigma, m, k_{y}, p}^{R},
\end{aligned}
$$




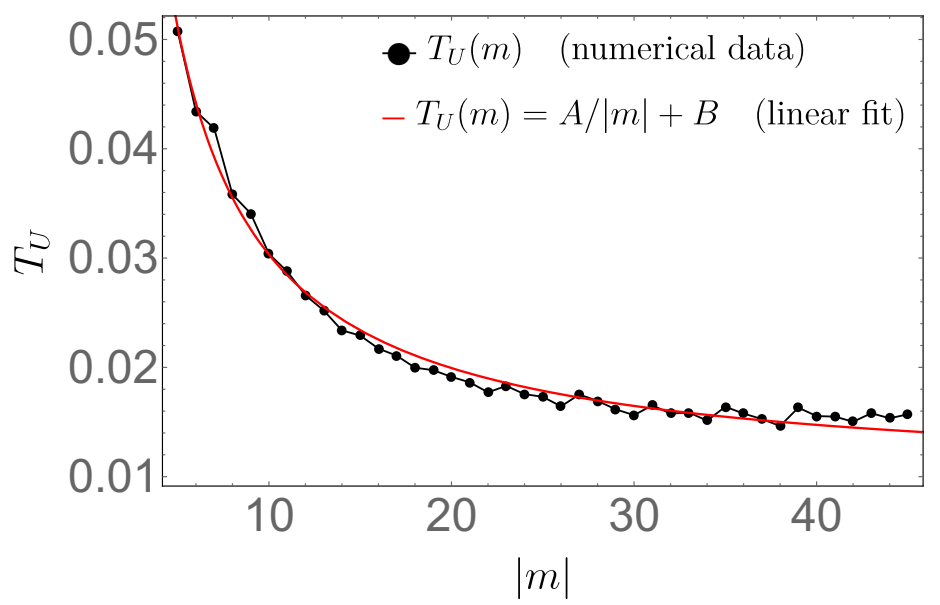

Figure 1: (color online) The Unruh temperature $T_{U}$ in a finite lattice system for the observer moving with a constant acceleration $|a|=1 /|m|$. We consider here a square lattice on a cylinder (open boundary conditions along $x$ ) of lengths $100 \times 100$, with the Rindler horizon laying on the circle along $y$ at the half of the cylinder. The numerical values $T_{U}(m)$ where found from fitting a continuum limit curve $(60)$ to the noninteracting numerical power spectrum for $\omega / \xi<1$. To the numerical results we fit $T_{U}(m)=A /|m|+B$ and find $A \approx 0.2$ and $B \approx 0.01$. Thus, the behavior of $T_{U}(m)$ in our finite lattice is remarkably close to the continuum result, $T_{U}(m)=1 /(2 \pi|m|)$, predicted by the Bisognano-Wichmann theorem.

and

$$
\begin{aligned}
& \Gamma_{k_{y}, p, p^{\prime}}^{(1)}=U_{k_{y}, p}^{R T} V_{-k_{y}, p^{\prime}}^{M}+V_{k_{y}, p}^{R T} U_{-k_{y}, p^{\prime}}^{M}, \\
& \Gamma_{k_{y}, p, p^{\prime}}^{(2)}=U_{-k_{y}, p}^{R \dagger} U_{-k_{y}, p^{\prime}}^{M}+V_{-k_{y}, p}^{R \dagger} V_{-k_{y}, p^{\prime}}^{M}
\end{aligned}
$$

Since we expand the Dirac fields in the eigenmodes of the Rindler Hamiltonian, we expect that the Fourier transform of (62) should express the power spectrum as seen by the accelerated observer $a=1 /|m|$. However, this statement is true only if $t$ is the proper time of the observer. In the Rindler Universe the proper time of an observer is $\xi_{m} t$, and consequently, to compare the response of different observers, the power spectrum of noninteracting particles needs to be rescaled as $\xi_{m} G_{m}\left(\omega / \xi_{m}\right)$ [100]. Furthermore, in the interacting problem we have a band gap separating valance and conductance bands. The band gap $2 \Delta_{R}(m)$ seen by a Minkowski observer is $|m|$ dependent. To account for that, we need to compare shifted power spectra, i.e. $\xi_{m} G_{m}\left(\left(\omega-\Delta_{R}(m)\right) / \xi_{m}\right)$.

Note that in the continuous limit, the Unruh temperature of noninteracting fermions is $T_{U}=1 /(2 \pi|m|)$. The numerical results for the finite lattice system show that indeed $T_{U}$ decreases with increasing $|m|$, but the functional dependence $T_{U}(m)$ might be in principle different. We investigate the relation $T_{U}(m)$ by fitting the continuum limit power spectrum (59) to the numerical results. Eventually, we find that $T_{U}(m)=A /|m|+B$ with $A \approx 0.2$ and $B \approx 0.01$ reproduces quite well the data for $5<|m|<45$, see Fig. 1. Thus, we find good agreement with the results of Bisognano-Wichmann theorem 115, 116] (see also [117]) that holds in the infinite lattice limit. Indeed, the theorem, which follows from axiomatic field theory, implies that all Lorentz-invariant local field theories display the Unruh effect with 

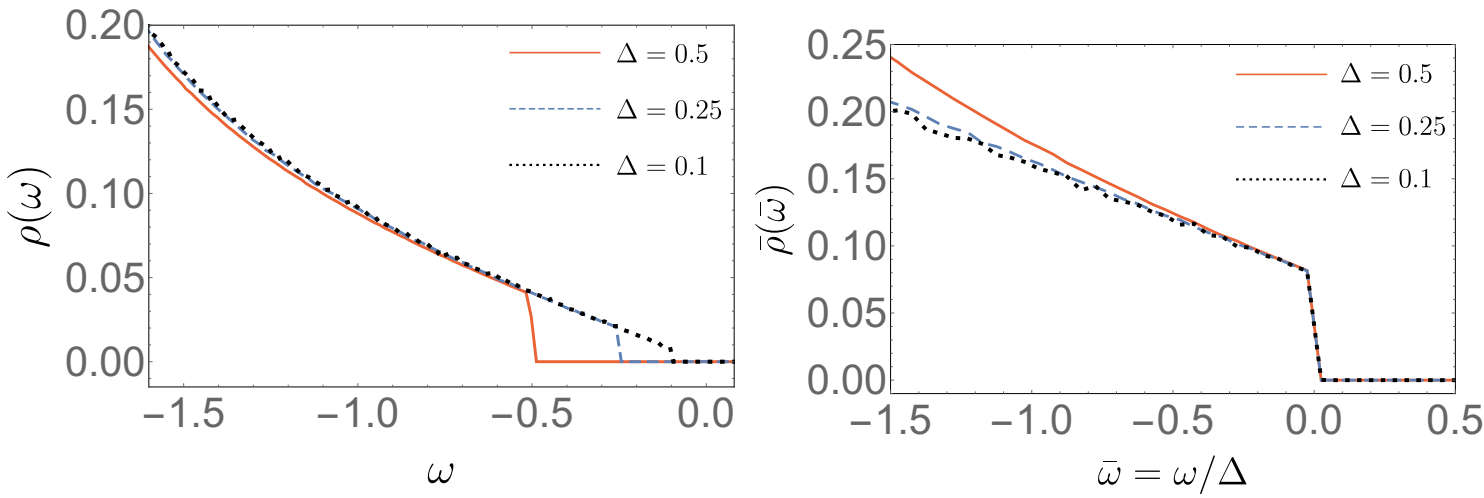

Figure 2: (color online) The numerical density of states $\rho(\omega)$ (left panel) and the rescaled densities $\bar{\rho}(\bar{\omega})$ (right panel) for a $\Delta$-paired Minkowski system on a torus with a quasiparticle dispersion relation (67). We consider here a square lattice on a torus (periodic boundary conditions along both $x$ and $y$ ) of lengths $5000 \times 5000$. For $|\omega|>\Delta$ the density of states is basically free fermionic. The rescaled density of states curves $\bar{\rho}(\bar{\omega})=\rho(\omega-\Delta) / \Delta$ overlap for $\bar{\omega} \approx 0$.

$T_{U}=1 /(2 \pi|m|)$. In the following we discuss in details the behavior of the Rindler noise in the different regimes of interest, extending the results obtained by us in 100 in the non-interacting Dirac fermions. As in 100 the numerical results are in the agreement with the continuum limit in the linear dispersion regime, i.e. $\omega / \xi<1$.

\subsection{Power spectrum at $T_{U} \approx 0$ and $T=0$}

We start by discussing the limit of zero acceleration, $T_{U} \rightarrow 0$, in which we expect the Wightman function in the Rindler space to coincide with the Wightman function in the Minkowski space at $T=0$.

By using the analytical solution for the Minkowski system on a torus (see Appendix A], we find for the latter

$$
G_{M}^{T=0}(t)=\left\langle 0_{M}\left|c_{\sigma, m, n}^{\dagger}(t) c_{\sigma, m, n}\right| 0_{M}\right\rangle=\frac{1}{2} \sum_{\vec{k}} e^{-i E_{\vec{k}} t}
$$

and consequently

$$
G_{M}^{T=0}(\omega)=\frac{1}{2} \sum_{\vec{k}} \delta\left(\omega+E_{\vec{k}}\right) \propto \rho(\omega)
$$

where

$$
E_{\vec{k}}=\sqrt{\Delta^{2}+4\left(\sin ^{2}\left(k_{x}\right)+\sin ^{2}\left(k_{y}\right)\right)},
$$

is a positive quasiparticle eigenenergy of a system and $\rho(\omega)$ is the negative energy density of states.

The numerical density of states $\rho(\omega)$ for different values of $\Delta$ is plotted on Fig. 2 (left panel). For the noninteracting system the density of states can be well approximated with 
the continuous limit density, i.e. $\rho(\omega) \propto|\omega|$ for $|\omega| \lesssim 1$. For the interacting system we can approximate

$$
G_{M}^{T=0}(\omega) \propto \rho(\omega)=\mathcal{N}^{-1}|\omega| \theta(-\omega-\Delta)
$$

for $|\omega| \lesssim 1$, i.e. a nonzero value of the pairing function $\Delta$ introduces a $2 \Delta$ band gap and a $\Delta$ step jump in the density of states. As $\Delta$ is much smaller than the half band width $w=2 \sqrt{2} \approx 2.83$ of the noninteracting system, for $|\omega| \gtrsim \Delta$ the density of state is basically free fermionic, and only at $|\omega| \approx \Delta$ encounters the step function jump. As we expect that $G_{M}^{T=0}(\omega)$ replicates the power spectrum of the Rindler noise for $T_{U}=0$, we can interpret this FermiDirac like behavior as a response of composite bosons (i.e. Cooper pairs), which should be fermionic (statistic inversion in even spatial dimensions). In other words the pairing influences power spectrum only near $|\omega| \approx \Delta$, which is expected as Cooper pairs tend to form near the Dirac cones (corresponding to $E_{\vec{k}} \approx \Delta$ ) and their number increases with $\Delta$ (see Appendix B).

Since we expect that (68) estimates the power spectrum of the Rindler noise for $T_{U}=0$ can infer that for $T_{U} \gtrsim 0$ the qualitative behavior of the power spectrum should be

$$
G(\omega) \propto \frac{|\omega|}{e^{(\omega+\Delta) / T_{U}}+1},
$$

for $|\omega| \lesssim 1$. It is trivial to realize that 69 in the limit $T_{U} \rightarrow 0$ recovers $(68)$. Also, for $\Delta \approx 0$ and $\omega / T_{U} \gg 1$ we can drop the plus one in denominator $(69)$ and therefore recover the power spectrum of noninteracting fermions 60 .

In order to compare the power spectra for different values of $\Delta$, we need to compensate for different densities of states of the interacting Minkowski ground states (a physical vacuum). After rescaling $\omega$ and shifting the argument of $\rho(\omega)$

$$
(\omega, \rho(\omega)) \rightarrow(\bar{\omega}, \bar{\rho}(\bar{\omega}))=(\omega / \Delta, \rho(\omega-\Delta) / \Delta),
$$

we find that all $\bar{\rho}(\bar{E})$ curves overlap near $\bar{E} \approx 0$, see Fig. 2 (right panel). Consequently, 69) after rescaling (70)

$$
\bar{G}(\bar{\omega})=\Delta^{-1} G(\omega-\Delta) \propto \frac{\bar{\omega}}{e^{\bar{\omega} /\left(T_{U} / \Delta\right)}+1},
$$

for $\bar{\omega} \approx 0$. Note that $\bar{G}(\bar{\omega})$ depends only on the ratio $\alpha=T_{U} / \Delta \propto 1 /(\Delta|m|)$, which might be interpreted as the effective Unruh temperature of the interacting accelerated gas. As a result, as long as $\alpha$ is constant, two nonequivalent accelerated observers might observe the same spectrum $\bar{G}(\bar{\omega})$ near $\bar{\omega} \approx 0$. This behavior is expected, since the interaction term of Rindler Hamiltonian (30) is invariant under the rescaling: $U \rightarrow U c$ and $|m| \rightarrow|m| / c$. Consequently, in the regime when interaction dominates over kinetic energy the power spectra $\bar{G}(\bar{\omega})$ with constant $|m| \Delta$ should coincide.

\subsection{Power spectrum at $T_{U}>0$ and $T=0$}

In this section we calculate the power spectra of the Rindler noise using the explicit formula (62) for the Wightman function. As discussed in Sec. 3.3, we rescale the power spectra according to the proper time of an observer $\tilde{G}(\tilde{\omega})=\xi_{m} G_{m}(\tilde{\omega}-\Delta)$, where $\tilde{\omega}=\omega / \xi_{m}$. The numerical results are presented on Fig. 3. where we plot the spectra $\tilde{G}(\tilde{\omega})$ for different pairing strengths $\Delta \approx 0.25$ (left panel), $\Delta \approx 0.5$ (right panel) and various distances to the horizon $|m|=10,20,30,40$. In order to both minimize lattice artifacts and to mimic realistic measurement schemes of finite space resolution, as in 100 we present the $\tilde{G}(\tilde{\omega})$ convoluted with a Gaussian filter of 2-site width. 

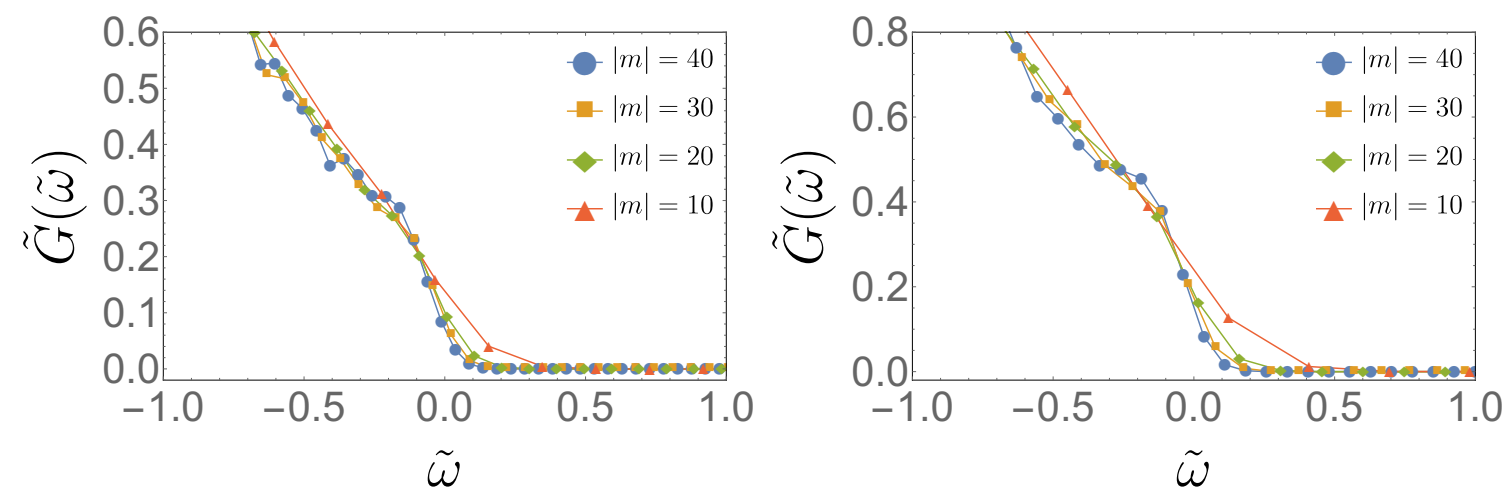

Figure 3: (color online) The power spectra of the Rindler noise, i.e. the Fourier transform of the Wightman function for the interacting Dirac fermions at $\mathrm{T}=0$ and the pairing function $\Delta \approx 0.25$ (left) $\Delta \approx 0.5$ (right). We consider here a square lattice on a cylinder (open boundary conditions along $x$ ) of lengths $100 \times 100$, with the Rindler horizon laying on the circle along $y$ at the half of the cylinder. For each value of the $|m|$, the $\tilde{G}(\tilde{\omega})$ are convoluted with a Gaussian filter of 2 -site width centered around $m$. For $\Delta \approx 0.25$ (left) the power spectrum is close to one of noninteracting fermions $(60)$, but with an indication of a Fermi-Dirac plateau. The plateau is more evident for $\Delta \approx 0.5$ (right), since the number of Cooper pairs becomes significant. The Fermi-Dirac profile is destroyed close to the horizon. Since the density of Cooper pairs is the greatest near the Dirac points, they dominate lowest energy excitations (close to $\tilde{\omega}=0$ ). For $|\tilde{\omega}| \gg 0$ the fermionic power spectrum is recovered. Note that the curves were rescaled to account for different proper times of different observers and shifted by $\Delta$ (due to the spectral gap).

The numerical results reproduce quite well the expected thermal behavior $(69)$ with the Unruh temperature inversely proportional to the distance to the horizon $T_{U} \propto 1 /|m|$. For $|\tilde{\omega}| \gg 0$ the power spectra are approximately linear (just like in a free fermionic case), while near $|\tilde{\omega}| \approx 0$ we observe a clear Fermi-Dirac profile (i.e. a bosonic response), which is more evident for $\Delta \approx 0.5$, since the number of Cooper pairs is more significant. Note that, as expected, the Fermi Dirac plateau is nearly twice as higher in for $\Delta \approx 0.5$ then in $\Delta \approx 0.25$.

After rescaling (71) we can directly compare different power-spectrum curves. The results are plotted on Fig. 4 . It turns out, that the postulated scaling is indeed valid near $|\tilde{\omega}| \approx 0$, see the discussion in the previous section.

Note that the chosen values of the pairing function $\Delta$ on Fig. 3 are one order of magnitude greater than the order of the Unruh temperature. For $\Delta \sim T_{U}$ we find that the power spectra are changed only marginally in comparison to the noninteracting ones.

\subsection{Power spectrum at $T_{U}>0$ and $T>0$}

In this section we again consider the power spectrum of Dirac fermions in the Rindler Universe, although, now we consider as background the thermal state at $T>0$ of the interacting Dirac Hamiltonian in Minkowski space. Such situation is relevant also from the experimental point of view as the ultracold fermions in optical lattices have typically a non-negligible temperature compared to the band width, thus order one in our units $(t=1)$. Thus, it is crucial to 


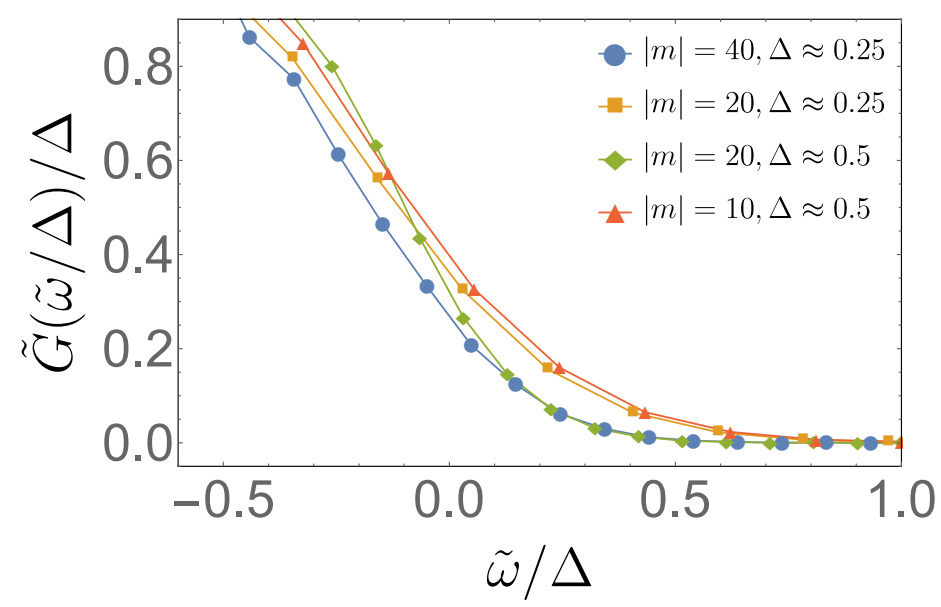

Figure 4: (color online) The power spectra rescaled to account for both proper-time difference of inequivalent observers and the difference in the density of states 70 . We consider here a square lattice on a cylinder (open boundary conditions along $x$ ) of lengths $100 \times 100$, with the Rindler horizon laying on the circle along $y$ at the half of the cylinder. For each value of the $|m|$, the $\tilde{G}(\tilde{\omega})$ are convoluted with a Gaussian filter of 2-site width centered around $m$. Because of the invariance of the interactions in Rindler Hamiltonian (30) under the rescaling, $U \rightarrow U c$ and $|m| \rightarrow|m| / c$ (see the discussion in the main text), we expect that when pairing dominates over kinetic energy, the power spectra with $|m| \Delta=$ constant, should be identical. Indeed, blue and green curves $(|m| \Delta=10)$, as well as red and orange curves $(|m| \Delta=5)$ overlap close to $\tilde{\omega} / \Delta=0$.

establish the interplay between the Unruh temperature $T_{U}$ and the physical temperature $T$ such to determine the visibility of the Unruh effect for interacting Dirac fermions, as done in 100 for the noninteracting case. The Wightman function can be written as

$$
G_{m}^{T}(t)=\operatorname{Tr}\left[\rho_{M}(T) c_{m, n}^{\dagger}(t) c_{m, n}\right]
$$

where

$$
\rho_{M}(T)=\sum_{k_{y}, p} n\left(E_{k_{y}, p}^{M}\right) \beta_{k_{y}, p}^{M \dagger}\left|0_{M}\right\rangle\left\langle 0_{M}\right| \beta_{k_{y}, p}^{M}
$$

and the power spectrum is

$$
G_{m}^{T}(\omega)=\int \mathrm{d} t e^{-i \omega t} G_{m}^{T}(t) .
$$

In order to compare with the results of the previous section, we choose the interaction $U=3.55$ and manipulate the physical temperature $T$ in such a way to obtain $\Delta(T) \approx 0.5,0.25$ (as in Fig. 3).

The numerical results are presented on Fig. 5. As expected, we find that the thermal background does not affect the Unruh effect until $\Delta(T) / T \sim 1$. In particular, for $T=0.315$ and $\Delta(T=0.315) \approx 0.25$ (left panel) we observe a thermal positive frequency contribution from the quasiparticles above the Fermi sea. For $T=0.1$ and $\Delta(T=0.1) \approx 0.5$ (right panel) the power spectrum is very close to the $T=0$ case. 

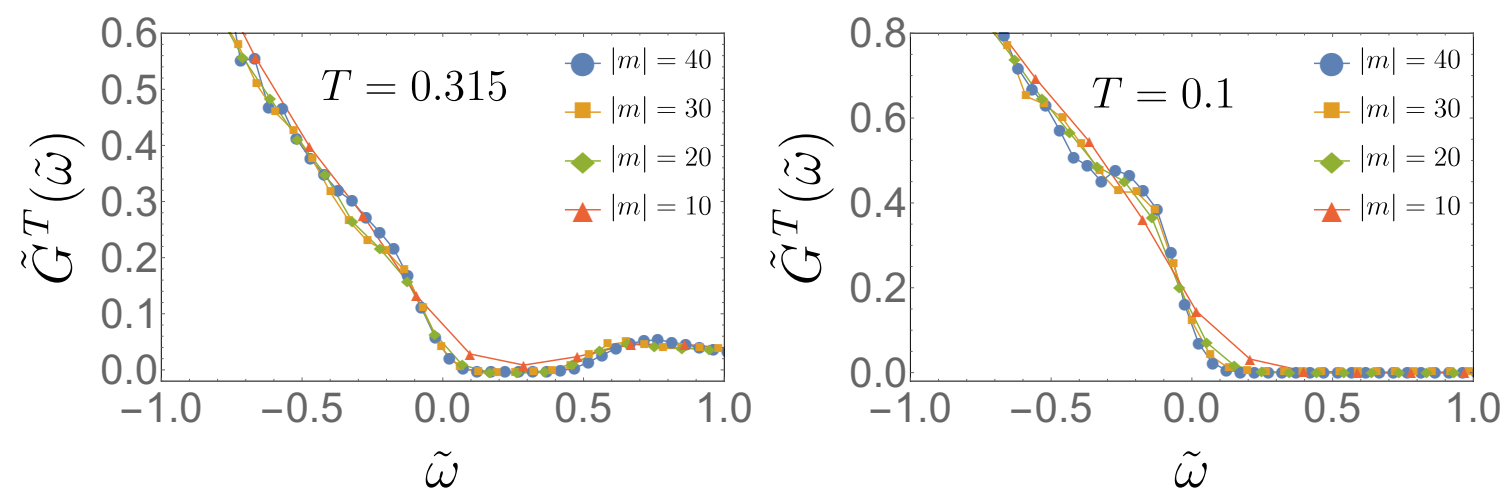

Figure 5: (color online) The power spectra of interacting Rindler Dirac fermions for a thermal background (74) at $T=0.315$ (left panel) and $T=0.1$ (right panel). We consider here a square lattice on a cylinder (open boundary conditions along $x$ ) of lengths $100 \times N_{y}$, with the Rindler horizon laying on the circle along $y$ at the half of the cylinder. For each value of the $|m|$, the $\tilde{G}(\tilde{\omega})$ are convoluted with a Gaussian filter of 2-site width centered around $m$. The temperature $T$ values were chosen such that $\Delta(T=0.315) \approx 0.25$ and $\Delta(T=0.1) \approx 0.5$ in order to directly compare with the results on Fig. 3 at $T=0$. As expected, the effect of a thermal background becomes more significant when the ratio $T / \Delta(T)$ becomes of order one.

\subsection{Experimental realization and detection of Unruh effect}

In [100] we presented a detailed proposal how to simulate the noninteracting Dirac Hamiltonian in Minkowski (35) and Rindler (48) spacetimes in the optical lattice setup. Since the tunneling matrices of a two component spinor $\psi_{m, n}$ is purely off-diagonal, then flipping its components at every second site $\psi_{m, n} \rightarrow \sigma_{x} \psi_{m, n}$ diagonalize the tunneling and the two components do not mix. Therefore, the noninteracting naive Dirac Hamiltonian on a square lattice is equivalent to two independent copies of a $\pi$-flux Hamiltonian, and the optical lattice simulation can be done with one component only. In other words, one can exploit that the lattice is bipartite and that the unit cell has dimension 2 due to the $\pi$ flux to encode the two components of Dirac spinor in two different sublattices (and reduce the doubling of Dirac points to 2). The key feature of our experimental proposal in [100] is that the tunneling is assisted in both $x$ and $y$ directions by Raman lasers that induce a synthetic magnetic field of flux $\pi$ in the symmetric gauge. The intensity of the tunneling is controlled by the intensity of (one of) Raman lasers. Such a scheme allows both for the preparation of the Minkowski vacuum as groundstate of the corresponding non-interacting Dirac Hamiltonian with uniform tunneling rates, and for its "acceleration" via a sudden quench of the tunneling rates to a $V$-shape profile of the Dirac Hamiltonian in Rindler spacetime.

The generalization of the scheme above to the case of interacting Dirac fermions requires the introduction of spatially tunable interactions. One possibility would be to realize the scheme in 100 with dipolar fermionic gases like erbium, where it has been very recently experimentally demonstrated that dipolar interactions are stable and tunable by Feshbach resonances [118]. In such a scheme, each of the two components of the spinor $c_{\uparrow}, c_{\downarrow}$ are identified with the two spin species of erbium that occupy two different sublattices. The interaction term $n_{\uparrow} n_{\downarrow}$, thus becomes a nearest-neighbor density-density interaction provided 
by the magnetic dipole moment of the atoms. The magnetic field (or in alternative the light shift) inducing the Feshbach resonance has to be then tuned spatially such to provide the desired $V$-shape interaction profile. In alternative, at the price of doubling the number of Dirac points, we can consider two spin states of fermionic atoms with Feshbach resonance like potassium [119 loaded in a spin-independent $\pi$-flux square lattice and perceiving the same laser-induced tunneling term. The interactions are now on site. A third possibility would be to incorporate in the set-up in [100] an additional lattice that hosts bosonic atoms that mediate the interaction between fermions in the same spin state as in 104. Again the interaction between the bosonic and fermionic species needs to possess a Feshbach resonance that allows to tune the scattering length appropriately.

In [100 we proposed an experimental scheme to measure $G(\omega)$ by using the one-particle excitation spectroscopy [120], which corresponds to a frequency-resolved transfer of the atoms to initially unoccupied auxiliary band of negligible width. In the weak-coupling limit the number of atoms transferred to the auxiliary band as a function of frequency detuning $\omega$ is proportional to $G(\omega)$. A similar scheme can be adopted here if the interactions in the auxiliary excited state are negligible.

\section{Pairing function $\Delta(U, T)$}

In Sec. 3.2 we argue that the pairing strength $\tilde{\Delta}_{R}=\xi_{m}^{-1} \Delta_{R}$ seen by an accelerated observer does not depend on the Unruh temperature, and is the same for all inequivalent observers in the Rindler Universe $\tilde{\Delta}_{R}=\Delta$. In this section we shall consider how the Minkowski pairing function $\Delta$ depends on the physical temperature $T$ and the interaction strength $|U|$.

At the half filling for the noninteracting system $U=0$, we have $\Delta=0$ and the ground state of the Minkowski Dirac Hamiltonian (23) is a Dirac semimetal with two valence and conduction bands touching at Dirac points $121-125$. Similarly to a standard BCS theory 113, 114, in a half-filled attractive Fermi Hubbard model on a square lattice even arbitrarily small attractive interactions $U<0$ give rise to a nonzero $\Delta$ pairing $[126 \sqrt{128}]$. On the contrary, it is known that below a critical interaction strength $\left|U_{c}\right| \neq 0$, Dirac fermions on a honeycomb lattice do not form Cooper pairs, since the density of states vanishes linearly at Dirac points 129 133. At $T=0$ and at the critical interaction $\left|U_{c}\right|$ the system undergoes the quantum phase transition between semimetal and a paired superconductor [134], although different methods, i.e. mean-field, variational and Monte Carlo give different estimates of the critical interaction $\left|U_{c}\right| \sim 2-5$. Note that honeycomb and square lattices are bipartite and therefore the particle-hole transformation allows us to relate attractive and repulsive Fermi Hubbard models at the half-filling [135].

We analyze $\Delta(U, T)$ dependence for two types of boundary conditions: (i) open in $x$ and periodic in $y$ (cylinder), (ii) periodic in both $x$ and $y$ (torus). We find that at $T=0$ the boundary conditions have strong affect the pairing properties of finite systems. In particular, we show that arbitrarily small $U<0$ gives rise to pairing in a small finite system on a torus.

\subsection{Solution on a cylinder}

Here we study in details the behavior of BCS pairing for the Hamiltonian (35) on a cylindrical square lattice. For concreteness, we present and discuss the numerical results for a cylinder of size $50 \times 50$ lattice system (open in $x$ direction). 

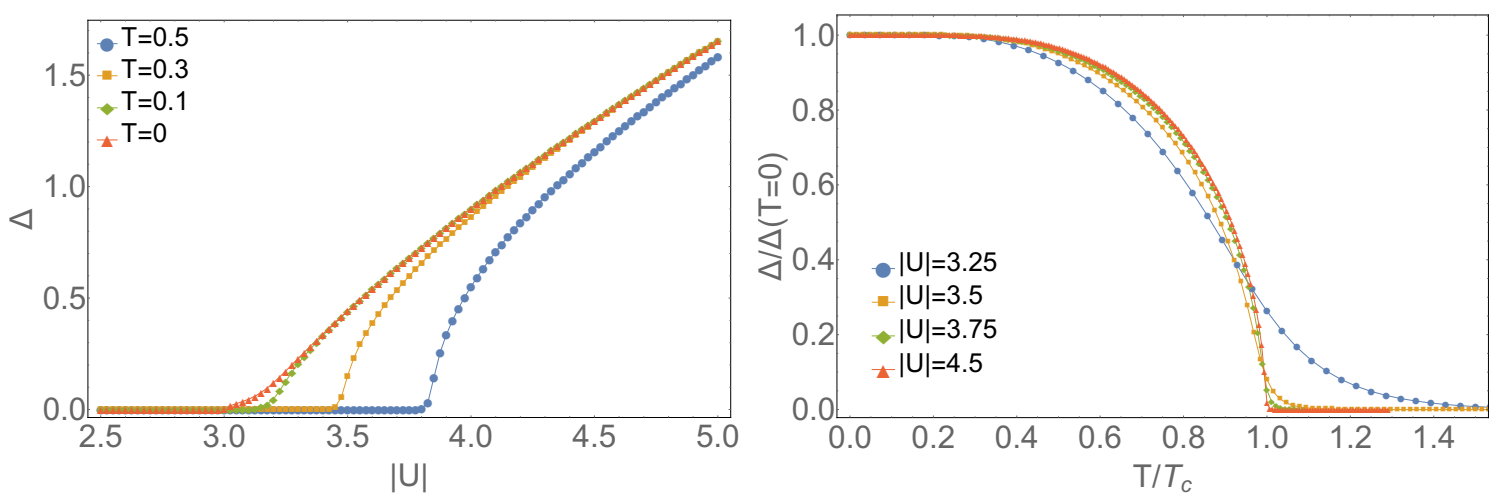

Figure 6: (color online) The meanfield pairing function $\Delta$ for a Dirac Hamiltonian (35) on a cylinder as a function of the interaction strength $|U|$ (left panel), and the temperature $T$ (right panel). We consider here a square lattice on a cylinder (open boundary conditions along $x$ ) of lengths $100 \times 100$. In particular, we find that: (i) $U_{c}$ increases with the temperature $T$ (left), (ii) the temperature phase transition is more evident when the interaction strength $|U|$ increases (right). Near $T=T_{c}$ for $U \gg U_{c}$ qualitatively recover the critial behavior known from the standard BCS theory, see the main text.

In Fig. 6 (left panel) we plot the pairing gap as a function of the interaction strength for several temperatures $T$. Our numerical results show that the pairing properties of the system described by the Hamiltonian (35) are similar to the Hubbard model on the honeycomb lattice, as one could expect. The quantitative differences might be due to the different dispersion relations away from the Dirac points. At $T=0$ we recover the critical value $\left|U_{c}\right| \approx 3$ as obtained for an attractive $\pi$-flux model [124]. For $T>0$, the critical interaction increases.

We plot the temperature dependence of the pairing function in Fig. 6 (right panel). As expected, we find that the pairing gap $\Delta(T)$ diminishes with the increasing temperature, and at some point we reach a normal unpaired state. In finite systems, it is always the crossover. Nevertheless, we observe that with increasing $U$ the behavior of $\Delta(T)$ starts to resemble a phase transition known from the standard BCS theory. It is known that in the standard BCS theory, the near critical behavior is universal [136]

$$
\Delta\left(T \approx T_{c}\right) \approx A \sqrt{1-T / T_{c}}
$$

and

$$
\Delta(T \approx 0) \approx B T_{c}
$$

where $A \approx 3.07 T_{c}, B \approx 1.764 T_{c}, A / B \approx 1.74$ are independent of material. We find that the numerical results on Fig. 6 (right panel) qualitatively reproduce the standard BCS theory, with 75 being a good approximation to the critical transition region. Quantitatively, the parameters $A$ and $B$ tend to the BCS values with increasing interaction strength $|U|$. In particular, we find $A \approx 2.40 T_{c}, B \approx 1.49 T_{c}, A / B \approx 1.62$ for $|U|=3.75$, and $A \approx 2.93 T_{c}$, $B \approx 1.68 T_{c}, A / B \approx 1.75$ for $|U|=4.5$. One may question the validity of the meanfield approach for values of the interactions of the order of the band width. While we can expect (small) quantitative deviations with respect to more precise approaches like diagrammatic quantum MonteCarlo, the qualitative behavior is known to be well captured by the mean field approach. 


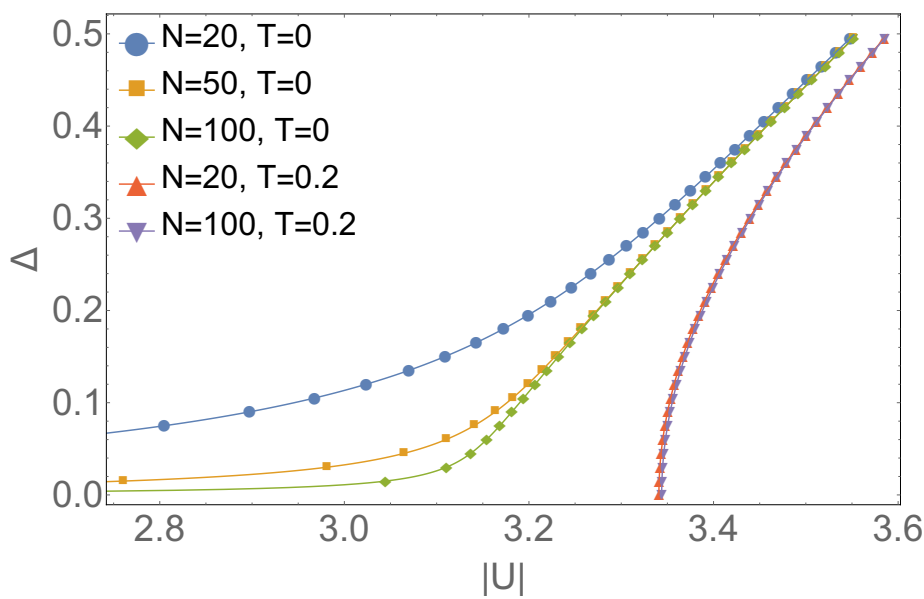

Figure 7: (color online) The meanfield pairing function $\Delta$ for a Dirac Hamiltonian (35) on a torus as a function of the interaction strength $|U|$ for different system sizes $N$ and temperatures $T$. We consider here a square lattice on a torus of lengths $N \times N$. The numerical results are in agreement with $(79)$. For $T \approx 0$ and $N=20$ the critical interaction is zero, but already for $N=100$ the pairing $\Delta(U)$ approaches the thermodynamic limit. For $T=0.2$ the finite size correction in 79 is negligible, the two curves for $N=20$ and $N=100$ overlap.

\subsection{Solution on a torus}

Here we analyze a system described by the Hamiltonian (35) in the meanfield approximation on a torus. Interestingly, in this case we find that for finite systems, the pairing does happen for arbitrarily small $|U|$ at $T=0$. We comment more on this point at the end of this section.

In this case the expression for $\Delta$ can be computed analytically (see the Appendix $\mathrm{A}$ for details) and reads

$$
\Delta=\frac{|U|}{N_{x} N_{y}} \sum_{\vec{k}} \frac{\Delta}{2 E_{\vec{k}}} \tanh \left(\frac{E_{\vec{k}}}{2 T}\right)
$$

where

$$
E_{\vec{k}}=\sqrt{\Delta^{2}+4\left(\sin ^{2}\left(k_{x}\right)+\sin ^{2}\left(k_{y}\right)\right)},
$$

is a positive quasiparticle eigenenergy of a system. From 77 we get

$$
\left|U_{c}(T)\right|=\lim _{\Delta \rightarrow 0+}\left(f(\Delta, T)+\frac{2}{\Delta N_{x} N_{y}} \tanh \left(\frac{\Delta}{2 T}\right)\right)^{-1}=\left(f(0, T)+\frac{1}{N_{x} N_{y} T}\right)^{-1}
$$

where

$$
f(\Delta, T)=\frac{1}{N_{x} N_{y}} \sum_{\vec{k} \neq D . P .} \frac{1}{2 E_{\vec{k}}} \tanh \left(\frac{E_{\vec{k}}}{2 T}\right),
$$

is finite in the thermodynamic limit $N_{x}, N_{y} \rightarrow \infty$. The summation in 80 goes over all $\vec{k}$ in the Brillouin zone except for $\vec{k} \in\{(00),(0 \pi),(\pi 0),(\pi \pi)\}$, since for $\Delta=0$ the dispersion relation has four Dirac points which require a separate treatment as $E_{\vec{k}}=0$. 
First of all, let us analyze the equation $(79)$ in thermodynamic limit. Taking $N_{x}, N_{y} \rightarrow \infty$ we get

$$
\left|U_{c}(T)\right| \stackrel{N \rightarrow \infty}{=} f(0, T)^{-1},
$$

which is finite for all $T<\infty$ and reproduces the numerical results for a system on a cylinder. Indeed, for $T \approx 0$ we obtain

$$
\left|U_{c}(T \approx 0)\right| \stackrel{N \rightarrow \infty}{=} f(0,0)^{-1} \approx 3.1
$$

Instead, for finite systems we have

$$
\left|U_{c}(T)\right| \stackrel{N<\infty}{=}\left(f(0, T)+\frac{1}{N_{x} N_{y} T}\right)^{-1},
$$

where now the second term cannot be dropped.

In particular, $\left|U_{c}(T)\right| \propto T$ for small $T$ and goes to zero in the limit $T \rightarrow 0+$. Note that if one puts $T=0$ in $(79)$ before taking the limit $\Delta \rightarrow 0+$ then the finite size result would be different.

Because $\lim _{T \rightarrow 0+}\left|U_{c}(T)\right|=0$, the pairing of Dirac fermions on torus can take place for an arbitrarily small interaction strength $|U|$. This apparent discrepancy between the two finite-size solutions of the Dirac Hamiltonian (23) with different boundary conditions can be explained as following. From 777 at $T=0$ the highest contribution to $\Delta$ comes from the smallest positive eigenvalues $E_{\vec{k}}$. In particular, for small $\Delta \approx 0$ the highest contribution comes from the Dirac cones $\vec{K} \in\{(00),(0 \pi),(\pi 0),(\pi \pi)\}$ (the density distribution of Cooper pairs is discussed in details in the Appendix $B$ which greatly affects small systems but is irrelevant in the thermodynamic limit, see Fig. 7. At the same time, this argument does not apply to the solution on the cylinder, as for even number of points $N$ the two bands touch only in a thermodynamic limit, $c f$. Fig. 6.

Different than for the naive Dirac Hamiltonian on a square lattice (23), the boundary conditions do not play a significant role for the honeycomb lattice, where the critical interactions is nonzero $\left|U_{c}\right|>0$ also on a torus. Again, such behavior admits a simple explanation in terms of the band structure of the model. The graphene dispersion relation

$$
E_{\vec{k}}=\sqrt{3+2 \cos \left(k_{y} \sqrt{3}\right)+4 \cos \left(\sqrt{3} / 2 k_{y}\right) \cos \left(3 / 2 k_{x}\right)+\Delta^{2}},
$$

is minimalized only in the thermodynamic limit as the Dirac cones' coordinates $\vec{K}= \pm(0,4 \pi /(3 \sqrt{3}))$ are not integer multiple of $\pi$. Thus, as it happens for finite cylindrical lattices the Dirac points do not contribute to the computation of $\Delta$ also in finite toroidal honeycomb lattices.

\section{Conclusions and Outlook}

In this paper we have investigated and proposed the experimental observation of the Unruh effect for interacting particles with ultracold fermions in optical lattices by a quantum quench. We have shown that achieving tunable Lorentz-preserving interactions with fermionic atoms is possible. Thus, it is possible to simulate an accelerated observer in an interacting background by simulating the corresponding Hamiltonian in Rindler space. Observing the Unruh effect

reduces then to measuring the Wightman response function in Rindler spacetime for the 
interacting background, the ground state of the interacting Dirac Hamiltonian in Minkowski space.

We have studied the Wightman response function detected by an accelerated observer for attractive relativistic interactions in the meanfield approximation, for varying interactions and real temperature $T$ of the background. In this approximation, the Unruh effect results from the interplay between the two different Bogoliubov transformations that relate the notion of particles for inertial and accelerated observers, and of particles and BCS quasi-particles, respectively. In the low-energy limit, in which the lattice system is with good approximation relativistic invariant, we have found that the Wightman function (precisely its power spectrum) displays the Planckian spectrum characteristic of the Unruh effect with a peculiarity. When the interactions grow, up to dominate over the Unruh temperature, there is a crossover between normal and "double" inversion of statistics determined by the (bosonic) Cooper pairs. Remarkably, in the low-energy limit our meanfield lattice calculations for interacting fermions not only give that the response is thermal, but also that the functional relation between the Unruh temperature $T_{U}$ and the proper acceleration $a$ is the same as for a free theory, $T_{U}=1 /(2 \pi a)$.

Such finding is in agreement with the predictions of the Bisognano-Wichmann theorem, which are valid under very general assumptions for any relativistic quantum field theory. On one hand, such fast convergence of lattice calculations to the correct relativistic behavior indicates that our lattice approach to the quantum simulation of quantum field theories in curved spacetime is promising. On the other hand, it can be read as a further evidence of the robustness of the Bisognano-Wichmann predictions recently observed in [137], where the equivalence between entanglement Hamiltonian and the Hamiltonian perceived by accelerated observers is used to access the entanglement spectrum of lattice models.

The present paper opens up interesting perspectives. It offers for instance a natural setup for testing quantum thermometry [138] and quantum thermodynamics [139 in curved spacetimes in presence of interactions (for a review on recent trends and developments in quantum thermodynamics see e.g. [140]).

It offers also a experimental playground for toy models of Lorentz-violating and transPlanckian physics [141]. Indeed, as we argue above there is a tight relation between Unruh effect and basic principles of relativistic quantum field theory as Lorentz invariance and locality. This tight relation explains the universality of the thermal behavior of the Wightman function [142, 143. Conversely, deviations from such behavior can signal e.g. the breaking of Lorentz invariance [144], the deformation of the uncertainty principle [145], or even open a window on quantum gravity [146]. Ultracold atom simulators of quantum interacting matter in artificial curved spacetime may serve for analyzing/testing such scenarios ( $c f$. with Lorentz violations in neutrino physics 147,148$]$ ).

Beyond the Unruh effect, another interesting direction for the quantum simulator we propose here is the study of dynamical chiral symmetry breaking in curved spacetime [149]. Indeed, by considering further atomic species we can in principle include more than one flavour and engineer the Gross-Neveu model [150] in an arbitrary optical (or more complicated) metric.

Last but not least, our study can be seen a further important step in the long journey to the simulation of self-gravitating quantum manybody physics. 


\section{Acknowledgements}

We thank Leticia Tarruell and Javier Rodríguez-Laguna for fruitful discussions.

Funding information A.K. acknowledges a support of the National Science Centre, Poland via Projects No. 2016/21/B/ST2/01086 and 2015/16/T/ST2/00504. M.L. acknowledges the Spanish Ministry MINECO (National Plan 15 Grant: FISICATEAMO No. FIS2016-79508-P, SEVERO OCHOA No. SEV-2015-0522), Fundació Cellex, Generalitat de Catalunya (AGAUR Grant No. 2017 SGR 1341 and CERCA/Program), ERC AdG OSYRIS, EU FETPRO QUIC, and the National Science Centre, Poland-Symfonia Grant No. 2016/20/W/ST4/00314. A.C. acknowledges financial support from the ERC Synergy Grant UQUAM and the SFB FoQuS (FWF Project No. F4016-N23)

\section{A Analytic equation for $\Delta$ on torus}

In this section we seek for a fully periodic solution of the mean-field Minkowski Hamiltonian at the half filling. After expressing the field operators in the momentum space, we can write

$$
H_{m f}^{M}=\frac{1}{2} \sum_{\vec{k}}\left(\begin{array}{ll}
\psi_{\vec{k}}^{\dagger} & \psi_{-\vec{k}}^{T}
\end{array}\right) H_{\vec{k}}^{M}\left(\begin{array}{c}
\psi_{\vec{k}} \\
\psi_{-\vec{k}}^{*}
\end{array}\right)
$$

where $\psi_{\vec{k}}^{\dagger}=\left(\begin{array}{cc}c_{\uparrow, \vec{k}}^{\dagger} & c_{\downarrow, \vec{k}}^{\dagger}\end{array}\right)$, and

$$
H_{\vec{k}}^{M}=\left(\begin{array}{cc}
\vec{g}_{\vec{k}} \circ \vec{\sigma} & i \Delta \sigma_{y} \\
-i \Delta \sigma_{y} & \vec{g}_{\vec{k}} \circ \vec{\sigma}^{*}
\end{array}\right)
$$

where $\Delta=|U|\left\langle c_{\uparrow, m, n} c_{\downarrow, m, n}\right\rangle, \vec{g}_{\vec{k}}=2 t\left(\sin \left(k_{x}\right) \sin \left(k_{y}\right)\right)$, and $\vec{\sigma}=\left(\sigma_{x} \sigma_{y}\right)$, such that $\vec{g}_{\vec{k}} \circ \vec{\sigma}=$ $2 t\left(\sin \left(k_{x}\right) \sigma_{x}+\sin \left(k_{y}\right) \sigma_{y}\right)$. The eigenvalues of $(86)$ read

$$
\lambda_{\vec{k}}= \pm \sqrt{\Delta^{2}+4\left(\sin ^{2}\left(k_{x}\right)+\sin ^{2}\left(k_{y}\right)\right)} \equiv \pm E_{\vec{k}} .
$$

The two eigenvectors associated to the positive eigenvalue are

$$
X_{\vec{k}, 1}^{(+)}=\frac{1}{\sqrt{2}}\left(\begin{array}{c}
1 \\
G_{\vec{k}} \\
0 \\
\frac{\Delta}{E_{\vec{k}}},
\end{array}\right), \quad X_{\vec{k}, 2}^{(+)}=\frac{1}{\sqrt{2}}\left(\begin{array}{c}
0 \\
-\frac{\Delta}{E_{\vec{k}}} \\
1 \\
G_{\vec{k}}^{*}
\end{array}\right),
$$

with $G_{\vec{k}}=\frac{2 t}{E_{\vec{k}}}\left(\sin \left(k_{x}\right)+i \sin \left(k_{y}\right)\right)$. Let us the write the field operator explicitly in terms of the eigenmodes

$$
\left(\begin{array}{c}
\psi_{\vec{k}} \\
\psi_{-\vec{k}}^{*}
\end{array}\right)=\sum_{p=1,2}\left(X_{\vec{k}, p}^{(+)} \beta_{\vec{k}, p}+X_{\vec{k}, p}^{(-)} \beta_{-\vec{k}, p}^{\dagger}\right),
$$

where $X_{\vec{k}, p}^{(-)}=\left(\sigma_{x} \otimes \mathbb{1}_{2}\right) X_{-\vec{k}, p}^{(+) *}$

Finally, by using the decomposition (89) we can obtain an equation for the pairing function

$$
\Delta=\frac{|U|}{N_{x} N_{y}} \sum_{\vec{k}}\left\langle c_{\uparrow, \vec{k}} c_{\downarrow, \vec{k}}\right\rangle=\frac{|U|}{N_{x} N_{y}} \sum_{\vec{k}} \frac{\Delta}{2 E_{\vec{k}}} \tanh \left(\frac{E_{\vec{k}}}{2 T}\right) .
$$




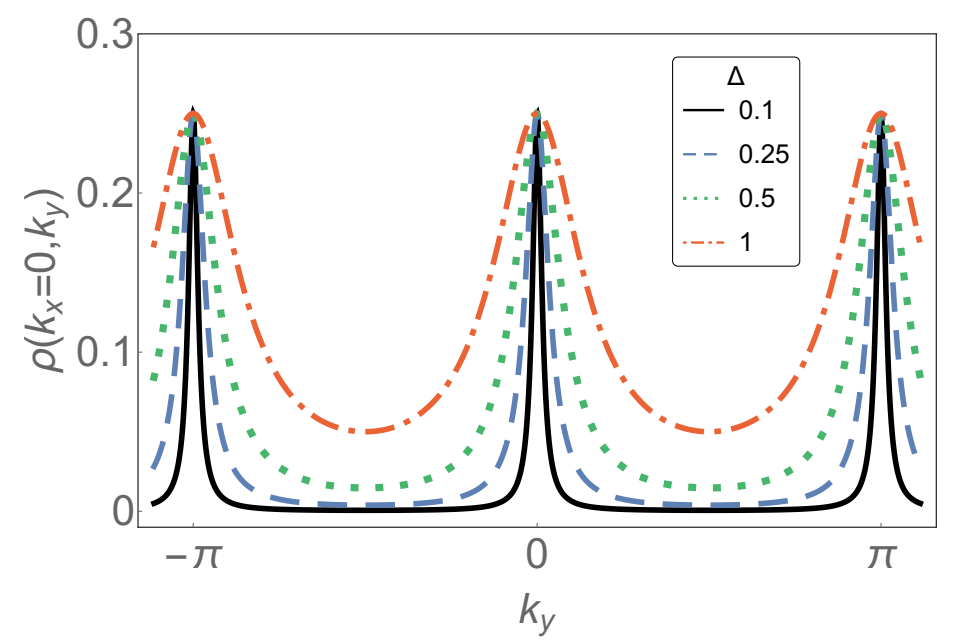

Figure 8: (color online) The plot of Cooper pairs density $\rho\left(k_{x}=0, k_{y}\right)$ obtained from (91). Up to $\Delta \approx 1$, with good approximation the Cooper pairs are formed around Dirac cones only.

\section{B Pairs density}

From (90), the quantity $\rho(\vec{k})=\left|\left\langle c_{\uparrow, \vec{k}} c_{\downarrow,-\vec{k}}\right\rangle\right|^{2}$ can be interpreted as the density of Cooper pairs in the whole system. Obviously, for $T=0$

$$
\rho(\vec{k})=\left|\left\langle c_{\uparrow, \vec{k}} c_{\downarrow,-\vec{k}}\right\rangle\right|^{2}=\frac{\Delta^{2}}{4 E_{\vec{k}}^{2}},
$$

where

$$
E_{\vec{k}}=\sqrt{\Delta^{2}+4\left(\sin ^{2}\left(k_{x}\right)+\sin ^{2}\left(k_{y}\right)\right)} .
$$

It is straightforward to conclude that for $\Delta \approx 0$ the density of Cooper pairs is very small $\rho(\vec{k}) \approx 0$, unless $E_{\vec{k}} \approx \Delta$. This is the case for the Dirac points $\vec{K} \in\{(00),(0 \pi),(\pi 0),(\pi \pi)\}$, where the density is maximized $\rho(\vec{K})=1 / 4$ and $E_{\vec{K}}=\Delta$.

This simple observation tells us that the pairing is dominated by Dirac-like excitations near the Dirac cones. This is the reason why the bosonic response (the Fermi Dirac plateau) only appears in the low frequency regime (Fig. 3).

We plot $\rho\left(k_{x}=0, k_{y}\right)$ in Fig. 8 . The figure shows that up to $\Delta \approx 1$, with good approximation the composite bosons (Cooper pairs) are formed around Dirac cones only.

\section{Symmetries of $H_{k_{y}}^{M / R}$}

In this part we consider all possible symmetries of the matrix Hamiltonian $H_{k_{y}}^{A}$, where $A$ stands both for $R$ (Rindler) or $M$ (Minkowski).

First of all, it is easy to find out that a unitary operator

$$
U_{1}=\sigma_{x} \otimes \mathbb{1}_{X} \otimes \mathbb{1}_{2}
$$


where $\mathbb{1}_{X}$ is the $N_{x} \times N_{x}$-identity matrix over the lattice coordinate $m$ along $x$, transforms the Hamiltonian as

$$
U_{1}^{\dagger} H_{k_{y}}^{A} U_{1}=-H_{-k_{y}}^{A *} .
$$

Thanks to this symmetry, we obtain in (46) and (52) the negative energy eigenmodes out of positive solutions $X_{\vec{k}, p}^{(-)}=U_{1} X_{-\vec{k}, p}^{(+) *}$.

Additionally, after making a gauge choice such that $\Delta \in \mathrm{R}$, then we see that there is another symmetry of the Hamiltonian

$$
U_{2}^{\dagger} H_{k_{y}}^{A} U_{2}=-H_{-k_{y}}^{A}
$$

where

$$
U_{2}=\sigma_{y} \otimes \mathbb{1}_{X} \otimes \sigma_{z}
$$

At the half filling, we also have

$$
U_{3}^{\dagger} H_{k_{y}}^{A} U_{3}=-H_{k_{y}}^{A *}
$$

where

$$
U_{3}=\sigma_{z} \otimes \mathbb{1}_{X} \otimes \mathbb{1}_{2} .
$$

All four possible combinations of $U_{1}, U_{2}, U_{3}$, i.e. $U_{1} U_{2}, U_{1} U_{3}, U_{2} U_{3}$ and $U_{1} U_{2} U_{3}$ are necessarily symmetries of $H_{k_{y}}^{A}$. In particular, defining $U_{4}=U_{1} U_{3}$ we see

$$
U_{4}^{\dagger} H_{k_{y}}^{A} U_{4}=H_{-k_{y}}^{A},
$$

therefore, we conclude $H_{k_{y}}^{A}$ and $H_{-k_{y}}^{A}$ have the same spectrum.

\section{References}

[1] R. P. Feynman, Int. J. Theor. Phys. 21, 467 (1982), doi:10.1007/BF02650179.

[2] I. Buluta and F. Nori, Science 326(5949), 108 (2009), doi:10.1126/science.1177838.

[3] I. M. Georgescu, S. Ashhab and F. Nori, Quantum simulation, Rev. Mod. Phys. 86, 153 (2014), doi:10.1103/RevModPhys.86.153.

[4] R. Blatt and C. F. Roos, Quantum simulations with trapped ions, Nature Physics 8(4), 277 (2012), doi::10.1038/nphys2252.

[5] K. R. Brown, J. Kim and C. Monroe, Co-designing a scalable quantum computer with trapped atomic ions, npj Quantum Information 2, 16034 (2016), doi:10.1038/npjqi.2016.34.

[6] M. Lewenstein, A. Sanpera and V. Ahufinger, Ultracold Atoms in Optical Lattices: Simulating Many-Body Quantum Systems, Oxford University Press, doi:10.1093/acprof:oso/9780199573127.001.0001 (2012).

[7] A. Celi, A. Sanpera, V. Ahufinger and M. Lewenstein, Quantum optics and frontiers of physics: the third quantum revolution, Physica Scripta 92(1), 013003 (2017), doi:10.1088/1402-4896/92/1/013003. 
[8] C. Gross and I. Bloch, Quantum simulations with ultracold atoms in optical lattices, Science 357(6355), 995 (2017), doi $10.1126 /$ science.aal3837.

[9] D. Jaksch and P. Zoller, Creation of effective magnetic fields in optical lattices: the hofstadter butterfly for cold neutral atoms, New J. Phys. 5(1), 56 (2003), doi 10.1088/1367$2630 / 5 / 1 / 356$.

[10] O. Boada, A. Celi, J. Latorre and M. Lewenstein, Quantum simulation of an extra dimension, Phys. Rev. Lett. 108(13), 133001 (2012), doi:10.1103/PhysRevLett.108.133001.

[11] A. Celi, P. Massignan, J. Ruseckas, N. Goldman, I. B. Spielman, G. Juzeliūnas and M. Lewenstein, Synthetic gauge fields in synthetic dimensions, Phys. Rev. Lett. 112(4), 043001 (2014), doi:10.1103/PhysRevLett.112.043001.

[12] M. Aidelsburger, M. Atala, M. Lohse, J. T. Barreiro, B. Paredes and I. Bloch, Realization of the hofstadter hamiltonian with ultracold atoms in optical lattices, Phys. Rev. Lett. 111, 185301 (2013), doi:10.1103/PhysRevLett.111.185301

[13] H. Miyake, G. A. Siviloglou, C. J. Kennedy, W. C. Burton and W. Ketterle, Realizing the harper hamiltonian with laser-assisted tunneling in optical lattices, Phys. Rev. Lett. 111, 185302 (2013), doi 10.1103/PhysRevLett.111.185302.

[14] M. Mancini, G. Pagano, G. Cappellini, L. Livi, M. Rider, J. Catani, C. Sias, P. Zoller, M. Inguscio, M. Dalmonte et al., Observation of chiral edge states with neutral fermions in synthetic hall ribbons, Science 349(6255), 1510 (2015), doi:10.1126/science.aaa8736.

[15] B. Stuhl, H.-I. Lu, L. Aycock, D. Genkina and I. Spielman, Visualizing edge states with an atomic bose gas in the quantum hall regime, Science 349(6255), 1514 (2015), doi:10.1126/science.aaa8515.

[16] A. Celi and L. Tarruell, Probing the edge with cold atoms, Science 349(6255), 1450 (2015), doi $10.1126 /$ science.aac7605.

[17] G. Jotzu, M. Messer, R. Desbuquois, M. Lebrat, T. Uehlinger, D. Greif and T. Esslinger, Experimental realization of the topological haldane model with ultracold fermions, Nature 515(7526), 237 (2014), doi:10.1038/nature13915.

[18] A. Eckardt, C. Weiss and M. Holthaus, Superfluid-insulator transition in a periodically driven optical lattice, Phys. Rev. Lett. 95(26), 260404 (2005), doi:10.1103/PhysRevLett.95.260404.

[19] P. Hauke, O. Tieleman, A. Celi, C. Ölschläger, J. Simonet, J. Struck, M. Weinberg, P. Windpassinger, K. Sengstock, M. Lewenstein et al., Non-abelian gauge fields and topological insulators in shaken optical lattices, Phys. Rev. Lett. 109(14), 145301 (2012), doi:10.1103/PhysRevLett.109.145301.

[20] A. Kosior and K. Sacha, Simulation of non-abelian lattice gauge fields with a singlecomponent gas, EPL (Europhysics Letters) 107(2), 26006 (2014).

[21] J. Struck, C. Ölschläger, R. Le Targat, P. Soltan-Panahi, A. Eckardt, M. Lewenstein, P. Windpassinger and K. Sengstock, Quantum simulation of frustrated classical magnetism in triangular optical lattices, Science 333(6045), 996 (2011), doi:10.1126/science.1207239. 
[22] A. Kosior and K. Sacha, Simulation of frustrated classical xy models with ultracold atoms in three-dimensional triangular optical lattices, Phys. Rev. A 87, 023602 (2013), doi:10.1103/PhysRevA.87.023602.

[23] A. Eckardt, Colloquium, Rev. Mod. Phys. 89, 011004 (2017), doi: $10.1103 /$ RevModPhys.89.011004.

[24] A. Mazurenko, C. S. Chiu, G. Ji, M. F. Parsons, M. Kanász-Nagy, R. Schmidt, F. Grusdt, E. Demler, D. Greif and M. Greiner, A cold-atom fermi-hubbard antiferromagnet, Nature 545(7655), 462 (2017), doi:10.1038/nature22362.

[25] F. Görg, M. Messer, K. Sandholzer, G. Jotzu, R. Desbuquois and T. Esslinger, Enhancement and sign change of magnetic correlations in a driven quantum many-body system, Nature 553(7689), 481 (2018).

[26] D. Mitra, P. T. Brown, E. Guardado-Sanchez, S. S. Kondov, T. Devakul, D. A. Huse, P. Schauss and W. S. Bakr, Quantum gas microscopy of an attractive fermi-hubbard system, Nature Physics 14(2), 173 (2018), doi:10.1038/nphys4297.

[27] A. Petrescu, M. Piraud, G. Roux, I. McCulloch and K. Le Hur, Precursor of the laughlin state of hard-core bosons on a two-leg ladder, Phys. Rev. B 96(1), 014524 (2017), doi:10.1103/PhysRevB.96.014524.

[28] M. C. Strinati, E. Cornfeld, D. Rossini, S. Barbarino, M. Dalmonte, R. Fazio, E. Sela and L. Mazza, Laughlin-like states in bosonic and fermionic atomic synthetic ladders, Phys. Rev. X 7(2), 021033 (2017), doi:10.1103/PhysRevX.7.021033.

[29] A. Celi, T. Grass, A. J. Ferris, B. Padhi, D. Raventós, J. Simonet, K. Sengstock and M. Lewenstein, Modified spin-wave theory and spin-liquid behavior of cold bosons on an inhomogeneous triangular lattice, Phys. Rev. B 94, 075110 (2016), doi:10.1103/PhysRevB.94.075110.

[30] K. G. Wilson, Confinement of quarks, Phys. Rev. D 10, 2445 (1974), doi: $10.1103 /$ PhysRevD.10.2445.

[31] L. Tagliacozzo, A. Celi, A. Zamora and M. Lewenstein, Optical abelian lattice gauge theories, Annals of Physics 330, 160 (2013), doi:10.1016/j.aop.2012.11.009.

[32] E. Zohar, J. I. Cirac and B. Reznik, Simulating compact quantum electrodynamics with ultracold atoms: Probing confinement and nonperturbative effects, Phys. Rev. Lett. 109(12), 125302 (2012), doi 10.1103/PhysRevLett.109.125302.

[33] D. Banerjee, M. Dalmonte, M. Müller, E. Rico, P. Stebler, U.-J. Wiese and P. Zoller, Atomic quantum simulation of dynamical gauge fields coupled to fermionic matter: From string breaking to evolution after a quench, Phys. Rev. Lett. 109, 175302 (2012), doi:10.1103/PhysRevLett.109.175302.

[34] S. Notarnicola, E. Ercolessi, P. Facchi, G. Marmo, S. Pascazio and F. V. Pepe, Discrete abelian gauge theories for quantum simulations of qed, J. Phys. A: Math. Theor 48(30), 30FT01 (2015), doi:10.1088/1751-8113/48/30/30FT01. 
[35] V. Kasper, F. Hebenstreit, M. Oberthaler and J. Berges, Schwinger pair production with ultracold atoms, Physics Letters B 760, 742 (2016), doi:10.1016/j.physletb.2016.07.036.

[36] O. Dutta, L. Tagliacozzo, M. Lewenstein and J. Zakrzewski, Toolbox for abelian lattice gauge theories with synthetic matter, Phys. Rev. A 95(5), 053608 (2017), doi: $10.1103 /$ PhysRevA.95.053608.

[37] D. González-Cuadra, E. Zohar and J. I. Cirac, Quantum simulation of the abelianhiggs lattice gauge theory with ultracold atoms, New J. Phys. 19(6), 063038 (2017), doi: $10.1088 / 1367-2630 /$ aa6f37.

[38] L. Tagliacozzo, A. Celi, P. Orland, M. Mitchell and M. Lewenstein, Simulation of nonabelian gauge theories with optical lattices, Nature Communications 4, 2615 (2013), doi: $10.1038 /$ ncomms3615.

[39] E. Zohar, J. I. Cirac and B. Reznik, Cold-atom quantum simulator for su (2) yang-mills lattice gauge theory, Phys. Rev. Lett. 110(12), 125304 (2013), doi:10.1103/PhysRevLett.110.125304.

[40] D. Banerjee, M. Bögli, M. Dalmonte, E. Rico, P. Stebler, U.-J. Wiese and P. Zoller, Atomic quantum simulation of $u(n)$ and $s u(n)$ non-abelian lattice gauge theories, Phys. Rev. Lett. 110(12), 125303 (2013), doi:10.1103/PhysRevLett.110.125303.

[41] E. A. Martinez, C. A. Muschik, P. Schindler, D. Nigg, A. Erhard, M. Heyl, P. Hauke, M. Dalmonte, T. Monz, P. Zoller et al., Real-time dynamics of lattice gauge theories with a few-qubit quantum computer, Nature 534(7608), 516 (2016), doi:10.1038/nature18318.

[42] C. Muschik, M. Heyl, E. Martinez, T. Monz, P. Schindler, B. Vogell, M. Dalmonte, P. Hauke, R. Blatt and P. Zoller, U (1) wilson lattice gauge theories in digital quantum simulators, New J. Phys. 19(10), 103020 (2017), doi:10.1088/1367-2630/aa89ab.

[43] L. Tagliacozzo, A. Celi and M. Lewenstein, Tensor networks for lattice gauge theories with continuous groups, Phys. Rev. X 4, 041024 (2014), doi:10.1103/PhysRevX.4.041024.

[44] M. C. Bañuls, K. Cichy, J. I. Cirac and K. Jansen, The mass spectrum of the schwinger model with matrix product states, JHEP 2013(11), 158 (2013), doi:10.1007/JHEP11(2013)158.

[45] B. Buyens, J. Haegeman, K. Van Acoleyen, H. Verschelde and F. Verstraete, Matrix product states for gauge field theories, Phys. Rev. Lett. 113(9), 091601 (2014), doi:10.1103/PhysRevLett.113.091601.

[46] E. Rico, T. Pichler, M. Dalmonte, P. Zoller and S. Montangero, Tensor networks for lattice gauge theories and atomic quantum simulation, Phys. Rev. Lett. 112(20), 201601 (2014), doi:10.1103/PhysRevLett.112.201601.

[47] P. Silvi, E. Rico, T. Calarco and S. Montangero, Lattice gauge tensor networks, New J. Phys. 16(10), 103015 (2014), doi:10.1088/1367-2630/16/10/103015.

[48] J. Haegeman, K. Van Acoleyen, N. Schuch, J. I. Cirac and F. Verstraete, Gauging quantum states: from global to local symmetries in many-body systems, Phys. Rev. X 5(1), 011024 (2015), doi:10.1103/PhysRevX.5.011024. 
[49] M. C. Bañuls, K. Cichy, J. I. Cirac, K. Jansen and H. Saito, Thermal evolution of the schwinger model with matrix product operators, Phys. Rev. D 92(3), 034519 (2015), doi:10.1103/PhysRevD.92.034519.

[50] E. Zohar, M. Burrello, T. B. Wahl and J. I. Cirac, Fermionic projected entangled pair states and local u (1) gauge theories, Annals of Physics 363, 385 (2015), doi:10.1016/j.aop.2015.10.009.

[51] U.-J. Wiese, Ultracold quantum gases and lattice systems: quantum simulation of lattice gauge theories, Annalen der Physik 525(10-11), 777 (2013), doi:10.1002/andp.201300104.

[52] E. Zohar, J. I. Cirac and B. Reznik, Quantum simulations of lattice gauge theories using ultracold atoms in optical lattices, Rep. Prog. Phys. 79(1), 014401 (2016), doi: $10.1088 / 0034-4885 / 79 / 1 / 014401$.

[53] M. Dalmonte and S. Montangero, Lattice gauge theory simulations in the quantum information era, Contemporary Physics 57(3), 388 (2016), doi: $10.1080 / 00107514.2016 .1151199$.

[54] O. Boada, A. Celi, J. I. Latorre and M. Lewenstein, New J.Phys. 13, 035002 (2011), doi:10.1103/PhysRevLett.108.133001.

[55] S. A. Fulling, Nonuniqueness of canonical field quantization in riemannian space-time, Phys. Rev. D 7, 2850 (1973), doi:10.1103/PhysRevD.7.2850.

[56] P. C. W. Davies, Scalar production in schwarzschild and rindler metrics, J. Phys. A: Math. Gen. 8(4), 609 (1975), doi:10.1088/0305-4470/8/4/022.

[57] S. W. Hawking, Particle creation by black holes, Commun. Math. Phys. 43(3), 199 (1975), doi:10.1007/BF02345020.

[58] W. G. Unruh, Notes on black-hole evaporation, Phys. Rev. D 14, 870 (1976), doi:10.1103/PhysRevD.14.870.

[59] N. D. Birrell and P. C. W. Davies, Quantum fields in curved space, 7. Cambridge University Press, doi:10.1016/j.physrep.2015.02.001 (1984).

[60] S. Takagi, Prog. Theor. Phys. Suppl. 88, 1 (1986), doi:10.1143/PTP.88.1.

[61] L. C. B. Crispino, A. Higuchi and G. E. A. Matsas, The unruh effect and its applications, Rev. Mod. Phys. 80, 787 (2008), doi:10.1103/RevModPhys.80.787.

[62] C. Barceló, S. Liberati and M. Visser, Analogue gravity, Living Reviews in Relativity 14(1), 3 (2011), doi:10.12942/lrr-2011-3.

[63] G. Volovik, The universe in a helium droplet, Oxford University Press, doi:10.1093/acprof:oso/9780199564842.001.0001 (2009).

[64] G. Gibbons, General Relativity, Cosmology and Astrophysics, chap. Some Links Between General Relativity and Other Parts of Physics, Springer International Publishing., doi:10.1007/978-3-319-06349-2_4 (2014). 
[65] L. J. Garay, J. R. Anglin, J. I. Cirac and P. Zoller, Sonic analog of gravitational black holes in bose-einstein condensates, Phys. Rev. Lett. 85, 4643 (2000), doi:10.1103/PhysRevLett.85.4643.

[66] P. O. Fedichev and U. R. Fischer, Gibbons-hawking effect in the sonic de sitter spacetime of an expanding bose-einstein-condensed gas, Phys. Rev. Lett. 91, 240407 (2003), doi: $10.1103 /$ PhysRevLett.91.240407.

[67] P. O. Fedichev and U. R. Fischer, Observer dependence for the phonon content of the sound field living on the effective curved space-time background of a bose-einstein condensate, Phys. Rev. D 69, 064021 (2004), doi:10.1103/PhysRevD.69.064021.

[68] P. O. Fedichev and U. R. Fischer, "cosmological" quasiparticle production in harmonically trapped superfluid gases, Phys. Rev. A 69, 033602 (2004), doi:10.1103/PhysRevA.69.033602.

[69] A. Retzker, J. I. Cirac, M. B. Plenio and B. Reznik, Methods for detecting acceleration radiation in a bose-einstein condensate, Phys. Rev. Lett. 101, 110402 (2008), doi:10.1103/PhysRevLett.101.110402.

[70] J.-C. Jaskula, G. B. Partridge, M. Bonneau, R. Lopes, J. Ruaudel, D. Boiron and C. I. Westbrook, Acoustic analog to the dynamical casimir effect in a bose-einstein condensate, Phys. Rev. Lett. 109, 220401 (2012), doi:10.1103/PhysRevLett.109.220401.

[71] D. Boiron, A. Fabbri, P.-E. Larré, N. Pavloff, C. I. Westbrook and P. Ziń, Quantum signature of analog hawking radiation in momentum space, Phys. Rev. Lett. 115, 025301 (2015), doi:10.1103/PhysRevLett.115.025301.

[72] J. Steinhauer, Observation of quantum hawking radiation and its entanglement in an analogue black hole, Nature Physics 12, 959 (2016), doi:10.1038/nphys3863.

[73] T. G. Philbin, C. Kuklewicz, S. Robertson, S. Hill, F. König and U. Leonhardt, Fiber-optical analog of the event horizon, Science 319(5868), 1367 (2008), doi:10.1126/science.1153625.

[74] F. Belgiorno, S. L. Cacciatori, M. Clerici, V. Gorini, G. Ortenzi, L. Rizzi, E. Rubino, V. G. Sala and D. Faccio, Hawking radiation from ultrashort laser pulse filaments, Phys. Rev. Lett. 105, 203901 (2010), doi:10.1103/PhysRevLett.105.203901.

[75] W. G. Unruh and R. Schützhold, Hawking radiation from "phase horizons" in laser filaments?, Phys. Rev. D 86, 064006 (2012), doi:10.1103/PhysRevD.86.064006

[76] S. Finazzi and I. Carusotto, Spontaneous quantum emission from analog white holes in a nonlinear optical medium, Phys. Rev. A 89, 053807 (2014), doi:10.1103/PhysRevA.89.053807.

[77] W. G. Unruh, Experimental black-hole evaporation?, Phys. Rev. Lett. 46, 1351 (1981), doi:10.1103/PhysRevLett.46.1351.

[78] S. Weinfurtner, E. W. Tedford, M. C. J. Penrice, W. G. Unruh and G. A. Lawrence, Measurement of stimulated hawking emission in an analogue system, Phys. Rev. Lett. 106, 021302 (2011), doi:10.1103/PhysRevLett.106.021302. 
[79] L. Feng, L. W. Clark, A. Gaj and C. Chin, Coherent inflationary dynamics for boseeinstein condensates crossing a quantum critical point, Nature Physics 14(3), 269 (2018), doi: $10.1038 / \mathrm{s} 41567-017-0011-\mathrm{x}$.

[80] S. Eckel, A. Kumar, T. Jacobson, I. B. Spielman and G. K. Campbell, A rapidly expanding bose-einstein condensate: An expanding universe in the lab, Phys. Rev. X 8, 021021 (2018), doi 10.1103/PhysRevX.8.021021.

[81] J. Minár and B. Grémaud, Mimicking dirac fields in curved spacetime with fermions in lattices with non-unitary tunneling amplitudes, J. Phys. A: Math. Theor 48(16), 165001 (2015), doi:10.1088/1751-8113/48/16/165001.

[82] C. Koke, C. Noh and D. G. Angelakis, Dirac equation in 2-dimensional curved spacetime, particle creation, and coupled waveguide arrays, Annals of Physics 374, 162 (2016), doi:10.1016/j.aop.2016.08.013.

[83] J. S. Pedernales, M. Beau, S. M. Pittman, I. L. Egusquiza, L. Lamata, E. Solano and A. del Campo, Dirac equation in $(1+1)$-dimensional curved spacetime and the multiphoton quantum rabi model, Phys. Rev. Lett. 120, 160403 (2018), doi:10.1103/PhysRevLett.120.160403.

[84] M. Cvetič, G. W. Gibbons and C. N. Pope, Photon spheres and sonic horizons in black holes from supergravity and other theories, Phys. Rev. D 94, 106005 (2016), doi:10.1103/PhysRevD.94.106005.

[85] G. Di Molfetta, M. Brachet and F. Debbasch, Quantum walks as massless dirac fermions in curved space-time, Phys. Rev. A 88(4), 042301 (2013).

[86] G. Di Molfetta, M. Brachet and F. Debbasch, Quantum walks in artificial electric and gravitational fields, Physica A: Statistical Mechanics and its Applications 397, 157 (2014), doi:10.1103/PhysRevA.88.042301.

[87] P. Arrighi, S. Facchini and M. Forets, Quantum walking in curved spacetime, Quantum Information Processing 15(8), 3467 (2016), doi:10.1007/s11128-016-1335-7.

[88] A. Mallick, S. Mandal, A. Karan and C. Chandrashekar, Simulating dirac hamiltonian in curved space-time by split-step quantum walk, arXiv preprint arXiv:1712.03911 (2017).

[89] A. Celi, Different models of gravitating dirac fermions in optical lattices, Euro. J. Phys. J. Special Topics 226(12), 2729 (2017), doi:10.1140/epjst/e2016-60390-y.

[90] L. Tarruell, D. Greif, T. Uehlinger, G. Jotzu and T. Esslinger, Creating, moving and merging dirac points with a fermi gas in a tunable honeycomb lattice, Nature 483(7389), 302 (2012), doi:10.1038/nature10871.

[91] P. Soltan-Panahi, J. Struck, P. Hauke, A. Bick, W. Plenkers, G. Meineke, C. Becker, P. Windpassinger, M. Lewenstein and K. Sengstock, Multi-component quantum gases in spin-dependent hexagonal lattices, Nature Physics 7(5), 434 (2011), doi:10.1038/nphys1916. 
[92] N. Fläschner, B. Rem, M. Tarnowski, D. Vogel, D.-S. Lühmann, K. Sengstock and C. Weitenberg, Experimental reconstruction of the berry curvature in a floquet bloch band, Science 352(6289), 1091 (2016), doi:10.1126/science.aad4568.

[93] L. Duca, T. Li, M. Reitter, I. Bloch, M. Schleier-Smith and U. Schneider, An aharonovbohm interferometer for determining bloch band topology, Science p. 1259052 (2014), doi: $10.1126 /$ science.1259052.

[94] F. de Juan, A. Cortijo and M. A. Vozmediano, Charge inhomogeneities due to smooth ripples in graphene sheets, Phys. Rev. B 76(16), 165409 (2007), doi: $10.1103 /$ PhysRevB.76.165409.

[95] A. Cortijo and M. A. Vozmediano, Effects of topological defects and local curvature on the electronic properties of planar graphene, Nuclear Physics B 763(3), 293 (2007), doi: $10.1016 /$ j.nuclphysb.2006.10.031.

[96] A. Iorio and G. Lambiase, The hawking-unruh phenomenon on graphene, Physics Letters B 716(2), 334 (2012), doi:10.1016/j.physletb.2012.08.023.

[97] M. Cvetič and G. W. Gibbons, Graphene and the zermelo optical metric of the btz black hole, Annals of Physics 327(11), 2617 (2012), doi:10.1016/j.aop.2012.05.013.

[98] A. Iorio and G. Lambiase, Quantum field theory in curved graphene spacetimes, lobachevsky geometry, weyl symmetry, hawking effect, and all that, Phys. Rev. D 90(2), 025006 (2014), doi:10.1103/PhysRevD.90.025006.

[99] M. Cariglia, R. Giambò and A. Perali, Curvature-tuned electronic properties of bilayer graphene in an effective four-dimensional spacetime, Phys. Rev. B 95, 245426 (2017), doi:10.1103/PhysRevB.95.245426.

[100] J. Rodriguez-Laguna, L. Tarruell, M. Lewenstein and A. Celi, Phys. Rev. A 95, 013627 (2017), doi:10.1103/PhysRevA.95.013627.

[101] W. E. Thirring, A soluble relativistic field theory, Annals of Physics 3(1), 91 (1958), doi:10.1016/0003-4916(58)90015-0.

[102] N. D. Birrell and P. C. W. Davies, Massless thirring model in curved space: Thermal states and conformal anomaly, Phys. Rev. D 18, 4408 (1978), doi: $10.1103 /$ PhysRevD.18.4408.

[103] C. Chin, R. Grimm, P. Julienne and E. Tiesinga, Feshbach resonances in ultracold gases, Rev. Mod. Phys. 82, 1225 (2010), doi:10.1103/RevModPhys.82.1225.

[104] J. I. Cirac, P. Maraner and J. K. Pachos, Cold atom simulation of interacting relativistic quantum field theories, Phys. Rev. Lett. 105, 190403 (2010), doi:10.1103/PhysRevLett.105.190403.

[105] L. Susskind, Phys. Rev. D 16, 3031 (1977), doi:10.1103/PhysRevD.16.3031.

[106] A. Messiah, Quantum Mechanics, vol. II, Dover Publications (1961).

[107] C. Itzykson and J.-B. Zuber, Quantum field theory, Courier Corporation (2006). 
[108] L. Parker and D. Toms, Quantum Field Theory in Curved Spacetime: Quantized Fields and Gravity, Cambridge University Press (2009).

[109] R. M. Wald, General Relativity, University of Chicago Press, Chicago, IL (1984).

[110] M. Soler, Phys. Rev. D 1, 2766 (1970), doi:10.1103/PhysRevD.1.2766.

[111] D. K. Hong and S. H. Park, Large-n analysis of the $(2+1)$-dimensional thirring model, Phys. Rev. D 49, 5507 (1994), doi:10.1103/PhysRevD.49.5507.

[112] S. Weinberg, The Quantum Theory of Fields, Cambridge University Press (1995).

[113] P. G. De Gennes, Superconductivity of Metals and Alloys, W. A. Benjamin, Inc., New York (1966).

[114] A. L. Fetter and J. D. Walecka, Quantum Theory of Many-Particle Systems, McGrawHill, New York (1971).

[115] J. J. Bisognano and E. H. Wichmann, On the duality condition for a hermitian scalar field, Journal of Mathematical Physics 16(4), 985 (1975), doi:10.1063/1.522605.

[116] J. J. Bisognano and E. H. Wichmann, On the duality condition for quantum fields, Journal of Mathematical Physics 17(3), 303 (1976), doi:10.1063/1.522898.

[117] G. L. Sewell, Quantum fields on manifolds: Pct and gravitationally induced thermal states, Annals of Physics 141(2), 201 (1982), doi:10.1016/0003-4916(82)90285-8.

[118] S. Baier, D. Petter, J. Becher, A. Patscheider, G. Natale, L. Chomaz, M. Mark and F. Ferlaino, Realization of a strongly interacting fermi gas of dipolar atoms, arXiv:1803.11445 (2018).

[119] J. S. Krauser, J. Heinze, S. Götze, M. Langbecker, N. Fläschner, L. Cook, T. M. Hanna, E. Tiesinga, K. Sengstock and C. Becker, Investigation of feshbach resonances in ultracold ${ }^{40}$ K spin mixtures, Phys. Rev. A 95, 042701 (2017), doi:10.1103/PhysRevA.95.042701.

[120] J. T. Stewart, J. P. Gaebler and D. S. Jin, Using photoemission spectroscopy to probe a strongly interacting fermi gas, Nature 454, 744 (2008), doi 10.1038/nature07172.

[121] P. R. Wallace, The band theory of graphite, Phys. Rev. 71, 622 (1947), doi:10.1103/PhysRev.71.622.

[122] K. Novoselov, A. Geim, S. Morozov, D. Jiang, M. Katsnelson, I. Grigorieva, S. Dubonos and A. Firsov, Two-dimensional gas of massless dirac fermions in graphene, Nature 438, 197 (2005), doi:10.1038/nature04233.

[123] A. H. Castro Neto, F. Guinea, N. M. R. Peres, K. S. Novoselov and A. K. Geim, The electronic properties of graphene, Rev. Mod. Phys. 81, 109 (2009), doi: $10.1103 /$ RevModPhys.81.109.

[124] G. Mazzucchi, L. Lepori and A. Trombettoni, J. Phys. B: At. Mol. Opt. Phys. 46, 134014 (2013), doi $10.1088 / 0953-4075 / 46 / 13 / 130201$. 
[125] J.-M. Hou, Moving and merging of dirac points on a square lattice and hidden symmetry protection, Phys. Rev. B 89, 235405 (2014), doi:10.1103/PhysRevB.89.235405.

[126] J. E. Hirsch, Two-dimensional hubbard model: Numerical simulation study, Phys. Rev. B 31, 4403 (1985), doi:10.1103/PhysRevB.31.4403.

[127] J. E. Hirsch and D. J. Scalapino, Enhanced superconductivity in quasi two-dimensional systems, Phys. Rev. Lett. 56, 2732 (1986), doi:10.1103/PhysRevLett.56.2732.

[128] R. T. Scalettar, E. Y. Loh, J. E. Gubernatis, A. Moreo, S. R. White, D. J. Scalapino, R. L. Sugar and E. Dagotto, Phase diagram of the two-dimensional negative-u hubbard model, Phys. Rev. Lett. 62, 1407 (1989), doi:10.1103/PhysRevLett.63.218.

[129] L. M. Martelo, M. Dzierzawa, L. Siffert and D. Baeriswyl, Mott-hubbard transition and antiferromagnetism on the honeycomb lattice, Zeitschrift für Physik B Condensed Matter 103(2), 335 (1996), doi:10.1103/PhysRevLett.97.230404.

[130] N. Furukawa, J. Phys. Soc. Jpn. 70, 1483 (2001), doi 10.1143/JPSJ.70.1483.

[131] T. Paiva, R. T. Scalettar, W. Zheng, R. R. P. Singh and J. Oitmaa, Groundstate and finite-temperature signatures of quantum phase transitions in the halffilled hubbard model on a honeycomb lattice, Phys. Rev. B 72, 085123 (2005), doi:10.1103/PhysRevB.72.085123.

[132] E. Zhao and A. Paramekanti, Bcs-bec crossover on the two-dimensional honeycomb lattice, Phys. Rev. Lett. 97, 230404 (2006).

[133] K. L. Lee, K. Bouadim, G. G. Batrouni, F. Hébert, R. T. Scalettar, C. Miniatura and B. Grémaud, Attractive hubbard model on a honeycomb lattice: Quantum monte carlo study, Phys. Rev. B 80, 245118 (2009), doi:10.1103/PhysRevB.80.245118.

[134] I. F. Herbut, Interactions and phase transitions on graphene's honeycomb lattice, Phys. Rev. Lett. 97, 146401 (2006), doi:10.1103/PhysRevLett.97.146401.

[135] A. F. Ho, M. A. Cazalilla and T. Giamarchi, Quantum simulation of the hubbard model: The attractive route, Phys. Rev. A 79, 033620 (2009), doi:10.1103/PhysRevA.79.033620.

[136] M. Tinkham, Introduction to Superconductivity, Dover Publications (1996).

[137] M. Dalmonte, B. Vermersch and P. Zoller, Quantum simulation and spectroscopy of entanglement hamiltonians, arXiv:1707.04455 (2017).

[138] S. Robles and J. Rodriguez-Laguna, Local quantum thermometry using unruh-dewitt detectors, Journal of Statistical Mechanics: Theory and Experiment 2017(3), 033105 (2017), doi:10.1088/1742-5468/aa60cd.

[139] E. Arias, T. R. de Oliveira and M. Sarandy, The unruh quantum otto engine, JHEP 2018(2), 168 (2018), doi:10.1007/JHEP02(2018)168.

[140] M. N. Bera, A. Riera, M. Lewenstein and A. Winter, Generalized laws of thermodynamics in the presence of correlations, Nature communications 8(1), 2180 (2017), doi: $10.1038 /$ s41467-017-02370-x. 
[141] P. Nicolini and M. Rinaldi, A minimal length versus the unruh effect, Physics Letters B 695(1-4), 303 (2011), doi $10.1016 /$ j.physletb.2010.10.051.

[142] N. Kajuri, Unruh effect in nonlocal field theories, Phys. Rev. D 95, 101701 (2017), doi:10.1103/PhysRevD.95.101701.

[143] L. Modesto, Y. S. Myung and S.-H. Yi, Universality of the unruh effect, Phys. Rev. D 97, 044016 (2018), doi:10.1103/PhysRevD.97.044016.

[144] R. Carballo-Rubio, L. J. Garay, E. Martin-Martinez and J. de Ramon, The unruh effect without thermality, arXiv:1804.00685 (2018).

[145] F. Scardigli, M. Blasone, G. Luciano and R. Casadio, Modified unruh effect from generalized uncertainty principle, arXiv:1804.05282 (2018).

[146] N. Alkofer, G. D'Odorico, F. Saueressig and F. Versteegen, Quantum gravity signatures in the unruh effect, Phys. Rev. D 94, 104055 (2016), doi:10.1103/PhysRevD.94.104055.

[147] V. A. Kostelecký and N. Russell, Data tables for lorentz and cpt violation, Rev. Mod. Phys. 83, 11 (2011), doi:10.1103/RevModPhys.83.11.

[148] Z. Lan, A. Celi, W. Lu, P. Öhberg and M. Lewenstein, Tunable multiple layered dirac cones in optical lattices, Phys. Rev. Lett. 107, 253001 (2011), doi:10.1103/PhysRevLett.107.253001.

[149] T. Inagaki, T. Muta and S. D. Odintsov, Dynamical symmetry breaking in curved spacetime, Progress of Theoretical Physics Supplement 127, 93 (1997), doi:10.1143/PTPS.127.93.

[150] D. J. Gross and A. Neveu, Dynamical symmetry breaking in asymptotically free field theories, Phys. Rev. D 10, 3235 (1974), doi:10.1103/PhysRevD.10.3235. 